\title{
Confiabilidade Assintótica de Sistemas
} Reparáveis

\section{Esteban Fernández Tuesta}

\author{
DISSERTAÇÃO APRESENTADA \\ $\mathrm{AO}$ \\ INSTITUTO DE MATEMÁTICA E ESTATÍSTICA \\ DA \\ UNIVERSIDADE DE SÃO PAULO \\ PARA OBTENÇÃO DO GRAU \\ $\mathrm{DE}$ \\ MESTRE EM ESTATÍSTICA
}

\section{Área de Concentração: Probabilidade Orientador: Prof. Dr. Vanderlei da Costa Bueno}

Durante a elaboração deste trabalho, o autor recebeu apoio financeiro da CAPES

-São Paulo, maio de 1999- 


\title{
Confiabilidade assintótica de sistemas reparáveis
}

\author{
Este exemplar corresponde à redação final \\ da dissertação devidamente corrigida e \\ defendida por Esteban Fernández Tuesta \\ e aprovada pela comissão julgadora.
}

São Paulo, 18 de junho de 1999.

Banca examinadora:

- Prof. Dr. Vanderlei da Costa Bueno (Orientador) - IME - USP

- Prof. Dr. Nikolai Kolev - IME - USP

- Prof. Dr. Osvaldo do Valle Costa - PEE - USP. 


\section{Resumo}

$\mathrm{Na}$ Teoria da Confiabilidade muitos problemas são resolvidos facilmente quando assumimos que todas as variáveis aleatórias envolvidas comportam-se de acordo com uma lei exponencial. Contudo as variáveis usadas nessa teoria tais como tempo de operação, tempo de reparo. etc., podem ter distribuições completamente diferentes. Neste caso usamos métodos de outras áreas tais como, Teoria das Filas e Teoria da Renovação, que permitem a solução de problemas quando parte ou todas as distribuições envolvidas são arbitrárias.

Outra forma é a procura de soluções aproximadas através de teoremas limite. Este trabalho segue esta linha.

\section{Abstract}

In Reliability Theory, many problems are solved in more simply form when we assume that all random variables involved have exponential law. But the random variables used in this theory like times of faultless operations, repair time and so on., can have completely different distributions. In this cases we use methods from another disciplines like Queueing theory and Renewal theory which permit the solution of problems when part or all distributions are arbitraries.

Another form of solution is to search for approximate formulas in the proofs of limit theorems. In this work, we follow this idea. 


\section{Agradecimentos}

A Deus, razão da minha vida.

Agradeço aos meus pais e irmãos.

Sou grato em especial ao meu orientador e amigo Prof. Dr. Vanderlei, pela sugestão do tema, pelos conselhos, pela nossa amizade, pela paciência ad infinitum durante o curso de Mestrado e orientação desta dissertação.

Aos grandes amigos que conheci no IME: Luis Gustavo (Corinthiano), José Flores, Gaspar, Roberto Madariaga, Delhi, Katia, Bety, Rodrigo, Lilian, Adriana, Sumaia entre outros.

Aos amigos da igreja: Alexandre, Cristina, Daniel, Elpidio, Hildebrando, Emmanuel, etc.

Á família Serpa.

Aos amigos de sempre: Rodolfo, Héctor, Fredy, Sixto, Noemí, Julia, Pedro, Marco Antonio, Jeanette, Janet, e muitos mais. 


\section{Sumário}

INTRODUÇÃO

1 Confiabilidade de sistemas com componentes reparáveis e distribuições exponenciais 4

1.1 Tempos de vida e de reparo com distribuições exponenciais . . . . . . . . . 5

1.1.1 Limites para a diferença entre a distribuição do tempo de vida do sistema e a distribuição exponencial . . . . . . . . . . . . . . . 6

1.1.2 Processos em estado estacionário . . . . . . . . . . . . . . 12

1.1.3 Tempo de Permanência nos estados de funcionamento . . . . . . . . 17

1.2 Tempos de reparo arbitrariamente distribuídos . . . . . . . . . . . . . . . 19

1.2.1 Aparecimento de um evento raro . . . . . . . . . . . . . . 20

1.2.2 Processo Regenerativo de duas fases . . . . . . . . . . . . . . . . 22

2 Confiabilidade de sistemas com componentes reparáveis e distribuições arbitrárias

2.1 Definições e Notações . . . . . . . . . . . . . . . . . . . . . . . . . . 26

2.2 Teorema Principal . . . . . . . . . . . . . . . . . . 30

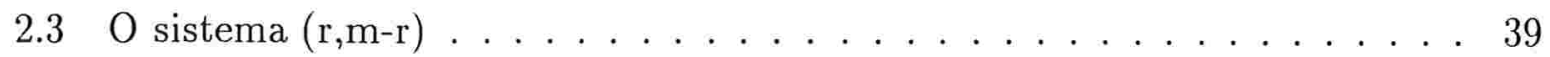

Apêndice 
A Lemas para o Processo regenerativo de duas fases

B Transformada de Laplace Racional

C Desigualdade de Chebyshev

D Lemas para o sistema (r,m-r)

E Processo de renovação a tempo discreto 


\section{INTRODUÇÃO}

A Teoria da Confiabilidade analisa numa abordagem probabilista, o funcionamento de objetos (sistemas ou componentes) no decorrer do tempo. Nesta dissertação, estudamos o comportamento de objetos reparáveis. Entenda-se por objeto reparável aquele que pode continuar determinada função após a eliminação da falha. Em alguns casos falamos de reparo completo, onde substituimos o objeto por um outro idêntico em perfeito funcionamento. Em particular, consideramos "reparo rápido", quando o tempo médio de reparo de um componente é muito menor que seu tempo médio de falha.

Como ilustração consideremos um sistema com dois componentes, um deles em operação e o outro em "standby". Quando o componente em operação falha, é substituído pelo componente em "standby" e encaminhado para reparo. Se o reparo é completado antes do componente em operação falhar, ele volta a substituí-lo e o componente, então em operação, volta ao "standby"; caso contrário o sistema falha. Seja $p, 0<p \ll 1$, muito pequena, a probabilidade de falha do sistema. Denominamos por um ciclo ao intervalo aleatório de tempo $\xi$ que começa quando colocamos o componente em operação e termina no fim de seu reparo. Sejam $\xi_{i}, i=1,2, \ldots$, cópias independentes identicamente distribuídas a $\xi$ e seja $\eta$ o intervalo aleatório que começa quando colocamos o componente em operação e termina quando o sistema falha. Na figura 0.1 mostramos um possível comportamento do sistema. Assim, se denotamos por $\tau$ o tempo de falha do sistema, podemos representá-lo por:

$$
\tau=\xi_{1}+\xi_{2}+\cdots+\xi_{N}+\eta,
$$


onde $N$ é uma variável aleatória que indica o número de ciclos precedentes à falha do sistema. Claramente, $N$ tem distribuição geométrica com parâmetro $p$, isto é:

$$
P(N=n)=(1-p)^{n} p, \quad n \geq 0
$$

Se consideramos $p \ll(1-p)$, o termo que domina em $\tau$ será

$$
\tau^{*}=\xi_{1}+\cdots+\xi_{N}
$$

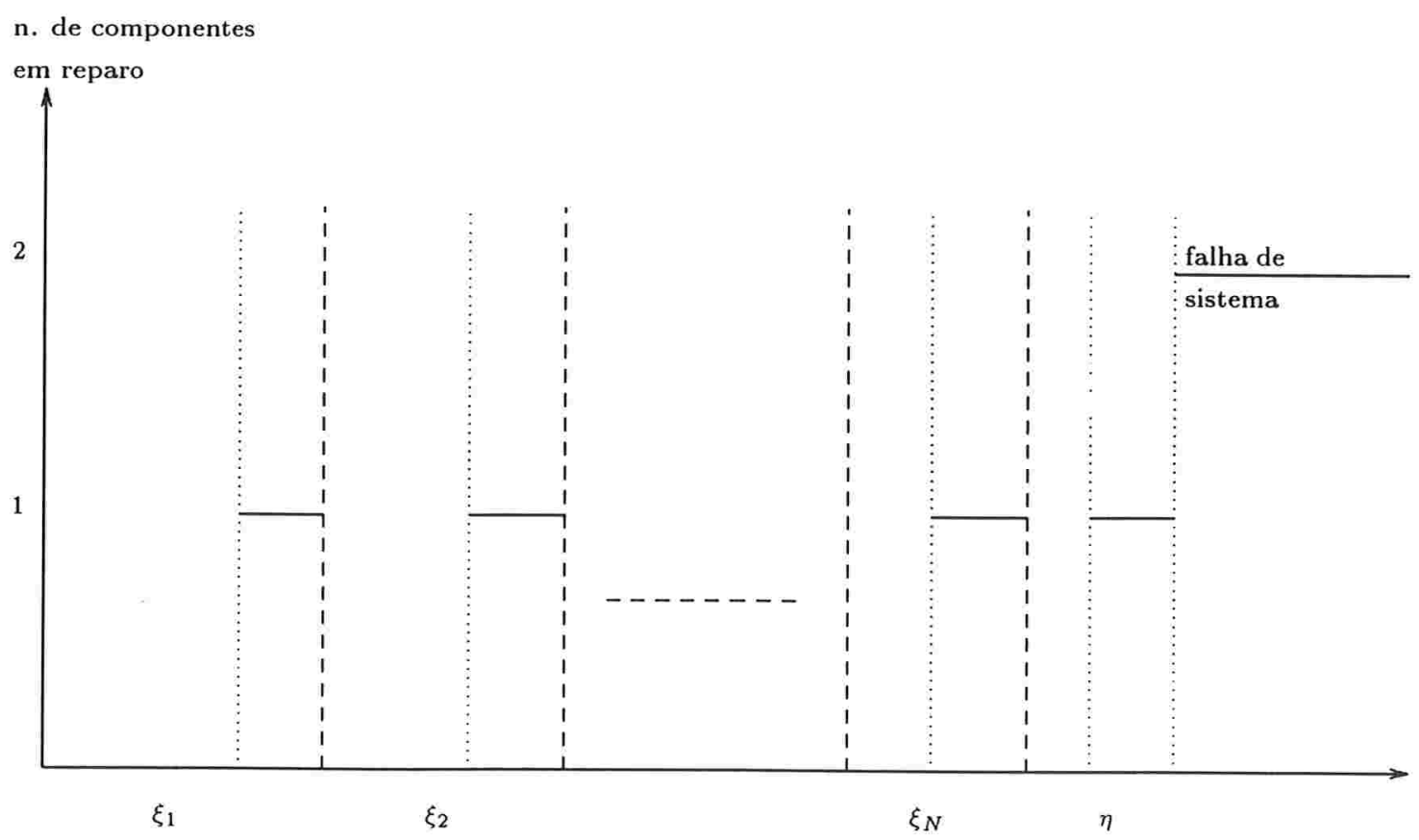

Figura 0.1 Esquema do tempo de vida do sistema

Se os $\xi_{i}, i=1,2, \ldots$ fossem constantes teriamos que $\tau^{*}$ é uma variável aleatória com distribuição geométrica, que sabemos, goza da propriedade de perda de memória, como a distribuição exponencial. Por outro lado, se os $\xi_{i}, i=1,2, \ldots$ fossem variáveis aleatórias independentes e identicamente distribuídas com distribuição exponencial, $\tau^{*}$ tería distribuição exponencial exata. Assim, de maneira heurística, é natural esperar que, sob condições um tanto gerais, a variável $\tau$, normalizada de maneira apropriada, se aproxime (em distribuição) da distribuição exponencial de parâmetro 1. 
Neste trabalho estudamos sistemas cujo funcionamento depende do número de componentes falhos no decorrer do tempo. No primeiro capítulo, analisamos o caso quando a distribuição dos tempos de vida e a distribuição dos reparos são exponenciais. Interpretamos o sistema como um Processo de Nacimento e Morte e delineamos os resultados propostos em [4]. Em seguida, analisamos o caso quando a distribuição dos tempos de vida são exponenciais e os tempos de reparo seguem uma distribuição arbitrária. No segundo capítulo, baseamos o estudo nos resultados propostos em [8] considerando que tanto os tempos de vida como os tempos de reparo dos componentes seguem uma distribuição arbitrária. Interpretamos o sistema como uma Fila e usamos técnicas desta teoria para obtermos os resultados mais importantes.

Resumimos algumas das suposições a serem feitas:

- Depois de reparado, o componente volta ao estado original.

- Cada unidade de manutenção pode reparar um elemento por vez.

- Os elementos não mudam de características quando estão no standby.

- O tempo para que um certo elemento falhe é, em média, muito menor que o tempo para a falha do sistema (os tempos de renovação são pequenos em comparação com os tempos de funcionamento de cada elemento). 


\section{Capítulo 1}

\section{Confiabilidade de sistemas com}

\section{componentes reparáveis e}

\section{distribuições exponenciais}

No decorrer desta dissertação estudamos a função de distribuição de tempos de vida de sistemas reparáveis em função das distribuições dos tempos de vida e reparo dos seus componentes. No presente capítulo. na seção 1, analisamos quando a distribuição do tempo de vida do sistema converge para uma distribuição exponencial. Em uma primeira abordagem, consideramos que os tempos de vida e os de reparo dos componentes têm funções de distribuições exponenciais e estudamos o sistema como um Processo de Nascimento e Morte. Aqui, um ciclo corresponde a um retorno ao estado zero sem cruzar um certo nível crítico $n$, que indica a falha do sistema. Consideramos também, o caso estacionário.

Na Seção 2, estudamos a convergência para a distribuição exponencial considerando um modelo um pouco mais geral, chamado Processo Regenerativo, analisando-o de duas maneiras: primeiro, quando há uma pequena probabilidade que a falha do sistema possa ocorrer em cada período e, segundo, quando dividimos um ciclo em duas partes: "períodos 
livres" e "períodos ocupados". O período livre corresponde ao funcionamento perfeito do sistema e seus componentes. O período ocupado descreve o comportamento do sistema quando alguns dos seus componentes estão sendo reparados. Sob as condições de reparo rápido, a probabilidade de ocorrer a falha do sistema nos períodos ocupados é muito pequena, o que implica que a variável aleatória $\tau$, que descreve o tempo de vida do sistema, é bastante similar a $\tau^{*}$ da introdução, isto é, $\tau$ é a soma aleatória de $N$ variáveis aleatórias exponenciais, onde $\mathrm{V}$ tem distribuição geométrica de parâmetro $p$, mais uma pequena parcela que não afeta o tipo da distribuição assintótica de $\tau$.

\subsection{Tempos de vida e de reparo com distribuições exponenciais}

Nesta seção, analisamos o tempo para a falha de sistemas reparáveis levando em consideração uma característica importante na manutenção de sistemas: o tempo médio de reparo de um componente é muitas vezes menor do que seu tempo médio de falha. Esta propriedade denominada "reparo rápido" no presente trabalho, permite avaliar o comportamento assintótico do tempo de vida do sistema. Algumas vezes podemos analisar o sistema como um processo de Nascimento e Morte onde o espaço de estados do sistema são os inteiros positivos e onde o estado $n$ representa a ocorrência de um evento indesejável: falha do sistema. Consideramos que os tempos de vida e de reparo dos componentes são variáveis aleatórias independentes e distribuídas com distribuições exponenciais de tal forma que a hipótese de reparo rápido seja satisfeita, isto é, os parâmetros dos tempos de vida são muito menores do que os parâmetros dos tempos de reparo. Neste caso, a distribuição do tempo de vida padronizado do sistema aproxima-se de uma distribuição exponencial de parâmetro um.

O estado do sistema em um instante determinado será representado pelo número de unidades falhadas naquele instante, constituíndo um Processo de Nascimento e Morte, 
onde $\left(\lambda_{k}\right)_{k=0}^{n}$ são as taxas de nascimento (chegadas ou falhas dos componentes), e $\left(\mu_{k}\right)_{k=0}^{n}$ são as taxas de morte (saídas ou reparos dos componentes). Consideramos que $n$, o estado de falha do sistema, é um estado absorvente, isto é, uma vez alcançado este estado, o sistema não pode ser reparado $\left(\lambda_{n}=0, \mu_{n}=0\right)$. Em particular, podemos pensar que o sistema funciona se ao menos $m$ das $M=m+n+1$ unidades estão em estado de operação, isto é, o mesmo processo pode ser interpretado como um sistema $m-o u t-o f-M$.

Se $\nu(t)$ é o número de componentes falhos no instante $t$, então

$$
\tau_{0, n}=\inf \{t: \nu(t)=n \mid \nu(0)=0\}
$$

indica o tempo de passagem do estado 0 para o estado $n, n \geq 1$, isto é, o tempo de vida do sistema.

Seja

$$
\xi=\frac{\tau_{0, n}}{\mathbb{E}\left[\tau_{0, n}\right]} .
$$

Quando o sistema é descrito por um Processo de Markov Homogêneo com um número finito de estados temos, conforme Solovyev (1972) (ver [4]), que se $x \in K_{0}$ (vide apêndice B), então a transformada de Laplace da variável aleatória $\xi$ pode ser expressa como:

$$
\mathbb{E}\left[e^{-z \xi}\right]=\left(1+z+a_{2} z^{2}+\cdots+a_{n} z^{n}\right)^{-1}=\prod_{k=1}^{n}\left(1+\alpha_{k} z\right)^{-1} .
$$

Pela Teoria de Polinômios Ortogonais, todas as raízes do polinômio acima são simples e negativas, isto é, os $\alpha_{k}$ são distintos e positivos.

\subsubsection{Limites para a diferença entre a distribuição do tempo de vida do sistema e a distribuição exponencial}

Dadas as observações acima, apresentamos um teorema provado por Solovyev (1972) que demonstra a convergência do tempo de vida do sistema para uma distribuição exponencial. 


\section{Teorema 1.1.1}

Se $\mathbb{E}\left[e^{-z \xi}\right]=\left(1+z+a_{2} z^{2}+\cdots+a_{n} z^{n}\right)^{-1}$, onde $a_{2}<\frac{1}{4}$,

então:

$$
\sup _{x \geq 0}\left|P(\xi>x)-e^{-x}\right|<\frac{1-\sqrt{1-4 a_{2}}}{1+\sqrt{1-4 a_{2}}}
$$

\section{PROVA}

Considerando que a Transformada de Laplace de uma variável aleatória com distribuição exponencial de parâmetro $1 / \alpha_{k}$ é $1 /\left(1+\alpha_{k} z\right)$ e que

$$
\mathbb{E}\left[e^{-z \xi}\right]=\left(1+z+a_{2} z^{2}+\cdots+a_{n} z^{n}\right)^{-1}=\prod_{k=1}^{n}\left(1+\alpha_{k} z\right)^{-1},
$$

deduzimos que

$$
\xi=\eta_{1}+\eta_{2}+\cdots+\eta_{n}
$$

onde os $\eta_{i}$ são variáveis aleatórias independentes e identicamente distribuídas com $\eta_{i} \sim$ $\exp \left(\frac{1}{\alpha_{i}}\right)$. Desta forma, o evento $\xi>x$ pode ocorrer de duas formas:

primeiro: $\eta_{1}>x$, então $\xi=\eta_{1}+\eta_{2}+\cdots+\eta_{n}>x$

segundo: $\eta_{1}=y$, onde $y \in(0, x)$ e $\eta_{2}+\eta_{3}+\cdots+\eta_{n}>x-y$.

Assim:

$$
\begin{aligned}
P(\xi>x) & =P\left(\eta_{1}+\eta_{2}+\cdots+\eta_{n}>x\right) \\
& =P\left(\eta_{1}>x\right)+\int_{0}^{x} P\left(\eta_{1}+\cdots+\eta_{n}>x \mid \eta_{1}=y\right) f_{\eta_{1}}(y) d y .
\end{aligned}
$$

Como $\eta_{1} \sim \exp \left(1 / \alpha_{1}\right)$, temos:

$$
\begin{aligned}
P(\xi>x) & =e^{-x / \alpha_{1}}+\int_{0}^{x} P\left(y+\eta_{2}+\cdots+\eta_{n}>x \mid \eta_{1}=y\right) \frac{1}{\alpha_{1}} e^{-y / \alpha_{1}} d y \\
& =e^{-x / \alpha_{1}}+\int_{0}^{x} P\left(\eta_{2}+\cdots+\eta_{n}>x-y \mid \eta_{1}=y\right) \frac{1}{\alpha_{1}} e^{-y / \alpha_{1}} d y .
\end{aligned}
$$

Dado que os $\eta_{i}$ são independentes, concluímos que

$$
P(\xi>x)=e^{-x / \alpha_{1}}+\int_{0}^{x} P\left(\eta_{2}+\cdots+\eta_{n}>x-y\right) \frac{1}{\alpha_{1}} e^{-y / \alpha_{1}} d y .
$$


Se fazemos $x-y=v, \quad-d y=d v$, teremos que

$$
P(\xi>x)=e^{-x / \alpha_{1}}+\int_{0}^{x} P\left(\eta_{2}+\cdots+\eta_{n}>v\right) \frac{1}{\alpha_{1}} e^{-(x-v) / \alpha_{1}} d v .
$$

Definindo

$$
\delta(x)=P(\xi>x)-e^{-x}
$$

temos:

$$
\delta(x)=e^{-x / \alpha_{1}}-e^{-x}+\int_{0}^{x} \frac{1}{\alpha_{1}} e^{-(x-v) / \alpha_{1}} P\left(\eta_{2}+\cdots+\eta_{n}>v\right) d v
$$

e concluímos que

$$
\delta(x) \geq e^{-x / \alpha_{1}}-e^{-x}
$$

pois o integrando é positivo.

Considere

$$
\psi(x)=e^{-x / \alpha_{1}}-e^{-x}, x>0 \text { onde } 0<\alpha_{1}<1 .
$$

Observe que $\psi(0)=0, \psi(\infty)=0$ e $\psi(x)<0$, para todo $x>0$. O ponto de mínimo da função é o ponto $x^{*}$ tal que $\psi^{\prime}\left(x^{*}\right)=0$ e $\psi^{\prime \prime}\left(x^{*}\right)>0$. Desta forma,

$$
\psi^{\prime}\left(x^{*}\right)=-\frac{e^{-x^{*} / \alpha_{1}}}{\alpha_{1}}+e^{-x^{*}}=0
$$

e portanto,

$$
e^{-x^{*}}=\frac{e^{-x^{*} / \alpha_{1}}}{\alpha_{1}}
$$

Note que,

$$
\psi^{\prime \prime}\left(x^{*}\right)=\frac{1}{\alpha_{1}} \frac{1}{\alpha_{1}} e^{-x^{*} / \alpha_{1}}-e^{-x^{*}}=\left(\frac{1}{\alpha_{1}}-1\right) e^{-x^{*}}>0
$$

Assim, $x^{*}$ satisfaz:

$$
\begin{aligned}
\frac{x^{*}}{\alpha_{1}}=x^{*} & -\log \alpha_{1}, \quad e \\
\psi(x) \geq \psi\left(x^{*}\right) & =e^{-x^{*} / \alpha_{1}}-e^{-x^{*}} \\
& =\alpha_{1} e^{-x^{*}}-e^{-x^{*}} \\
& =-e^{-x^{*}}\left(1-\alpha_{1}\right) .
\end{aligned}
$$


Como $x>0$ e $0<\alpha_{1}<1$, temos que $1-\alpha_{1}>0,0<e^{-x^{*} / \alpha_{1}}<1$, e segue de (1.6) que

$$
-e^{-x^{*}}>-\frac{1}{\alpha_{1}}
$$

Portanto, concluímos que:

$$
\delta(x) \geq \psi(x) \geq \psi\left(x^{*}\right)>-\frac{1-\alpha_{1}}{\alpha_{1}}=-\frac{\alpha}{1-\alpha},
$$

onde $\alpha=1-\alpha_{1}$. Por outro lado, por (1.4) e do fato que $e^{-x / \alpha_{1}}-e^{-x}<0$, temos:

$$
\begin{aligned}
\delta(x)=P(\xi>x)-e^{-x} & \leq \int_{0}^{x} \frac{1}{\alpha_{1}} e^{-(x-v) / \alpha_{1}} P\left(\eta_{2}+\cdots+\eta_{n}>v\right) d v \\
& \leq \int_{0}^{\infty} \frac{1}{\alpha_{1}} P\left(\eta_{2}+\cdots+\eta_{n}>v\right) d v \\
& =\frac{\mathbb{E}\left[\eta_{2}+\cdots+\eta_{n}\right]}{\alpha_{1}} \\
& =\frac{\alpha_{2}+\cdots+\alpha_{n}}{\alpha_{1}}
\end{aligned}
$$

pois os $\eta_{i}$ são variáveis aleatórias independentes e identicamente distribuídas com $\eta_{i} \sim$ $\exp \left(1 / \alpha_{i}\right)$.

Considerando a relação:

$$
1+z+a_{2} z^{2}+\cdots+a_{n} z^{n}=\prod_{k=1}^{n}\left(1+\alpha_{k} z\right)
$$

onde os $\alpha_{k}$ são distintos e positivos e igualando os coeficientes para $z$ e $z^{2}$ de ambos os lados da equação acima, obtemos:

$$
\begin{gathered}
\alpha_{1}+\alpha_{2}+\cdots+\alpha_{n}=1, \quad e \\
a_{2}=\sum_{i<j} \alpha_{i} \alpha_{j} .
\end{gathered}
$$

Assim,

$$
\delta(x) \leq \frac{1-\alpha_{1}}{\alpha_{1}}=\frac{\alpha}{1-\alpha}
$$

De (1.7) e (1.10) temos que

$$
|\delta(x)| \leq \frac{\alpha}{1-\alpha}
$$


Como

$$
\left(\sum_{i=1}^{n} \alpha_{i}\right)^{2}=\alpha_{1}^{2}+\alpha_{2}^{2}+\cdots+\alpha_{n}^{2}+2 \sum_{i<j} \alpha_{i} \alpha_{j}
$$

obtemos

$$
a_{2}=\sum_{i<j} \alpha_{i} \alpha_{j}=\frac{\left(\sum_{i=1}^{n} \alpha_{i}\right)^{2}-\sum_{i=1}^{n} \alpha_{i}^{2}}{2} .
$$

Das últimas equações concluímos que

$$
1-2 a_{2}=\alpha_{1}^{2}+\alpha_{2}^{2}+\cdots+\alpha_{n}^{2}
$$

Considerando, sem perda de generalidade, que $\alpha_{1}>\alpha_{2}>\ldots>\alpha_{n}$ e $\alpha=1-\alpha_{1}$ temos

$$
1-2 a_{2}=\alpha_{1} \alpha_{1}+\cdots+\alpha_{n} \alpha_{n}<\alpha_{1}\left(\alpha_{1}+\alpha_{2}+\cdots+\alpha_{n}\right)=\alpha_{1}=1-\alpha .
$$

Como $a_{2}<\frac{1}{4}$, temos $\frac{1}{2}<1-2 a_{2}<\alpha_{1}=1-\alpha$, e portanto $\alpha<\frac{1}{2}$.

Assim, de (1.13) segue que

$$
\begin{aligned}
1-2 a_{2} & <\alpha_{1}^{2}+\left(\alpha_{2}+\alpha_{3}+\cdots+\alpha_{n}\right)^{2} \\
& =\alpha_{1}^{2}+\left(1-\alpha_{1}\right)^{2} \\
& =(1-\alpha)^{2}+\alpha^{2}
\end{aligned}
$$

isto é,

$$
\alpha^{2}-\alpha+a_{2}>0
$$

A equação $\alpha^{2}-\alpha+a_{2}=0$ tem como soluções $\alpha=\frac{1 \pm \sqrt{1-4 a_{2}}}{2}$ (positivas). Contudo o fato que $\alpha<\frac{1}{2}$ implica

$$
\alpha<\frac{1-\sqrt{1-4 a_{2}}}{2} .
$$

Logo,

$$
|\delta(x)|<\frac{\alpha}{1-\alpha}<\frac{1-\sqrt{1-4 a_{2}}}{1+\sqrt{1-4 a_{2}}}
$$




\section{OBSERVAÇÃO 1.1}

Observe-se que se $a_{2} \rightarrow 0$ então,

$$
|\delta(x)|<\frac{\alpha}{1-\alpha}<\frac{1-\sqrt{1-4 a_{2}}}{1+\sqrt{1-4 a_{2}}} \rightarrow 0 .
$$

Assim,

$$
\xi=\frac{\tau_{0, n}}{\mathbb{E}\left[\tau_{0, n}\right]} \stackrel{d}{\longrightarrow} \exp (1)
$$

Logo, uma condição suficiente para a convergência é $a_{2} \rightarrow 0$.

\section{OBSERVAÇÃO 1.2}

No caso do Processo de Nascimento e Morte com parâmetros $\lambda_{k}$, $\mu_{k}$, temos:

$$
a_{2}=\frac{\sum_{k=1}^{n-1} \frac{1}{\lambda_{k} \theta_{k}} \sum_{s=1}^{k} \mu_{0, s} \theta_{s}}{\left(\sum_{k=0}^{n-1} \frac{\sum_{i=0}^{k} \theta_{i}}{\lambda_{k} \theta_{k}}\right)^{2}},
$$

onde

$$
\begin{gathered}
\theta_{0}=1 \\
\theta_{j}=\frac{\lambda_{0} \lambda_{1} \ldots \lambda_{j-1}}{\mu_{1} \mu_{2} \ldots \mu_{j}}, e \\
\mathbb{E}\left[\tau_{0, s}\right]=\mu_{0, s}=\sum_{k=0}^{s-1} \frac{\sum_{i=0}^{k} \theta_{i}}{\lambda_{k} \theta_{k}}
\end{gathered}
$$

$\operatorname{Ver}([1]$ pag 353-358)

\section{OBSERVAÇÃO 1.3}

Como foi dito no inicío do capítulo, $\lambda$ é menor que $\mu$. Se assumirmos agora que num processo de nascimento e morte podemos expressar $\lambda_{k}=\tilde{\lambda} \alpha$, com $\alpha \rightarrow 0$, substituindo então nas fórmulas para $\theta_{k}$, concluímos que $a_{2}=O\left(\alpha^{n}\right)$. 


\subsubsection{Processos em estado estacionário}

No Teorema 1.1.1 foi apresentado um limite para a diferença entre $P(\xi>x)$ e $e^{-x}$, onde $\xi=\frac{\tau_{0, n}}{E\left[\tau_{0, n}\right]}$. Nesta seção assumimos o Processo de Nascimento e Morte em estado estacionário, isto é, o sistema tem sido observado por um longo período de tempo e a probabilidade de atingir determinado estado é invariante. O sistema falha quando o processo atinge o nível $n$. No que segue, denotaremos por $T_{1}, T_{2}, \ldots$ os intervalos aleatórios dos sucessivos retornos ao estado zero, os quais são independentes e identicamente distribuídos com $\mu=\mathbb{E}\left[T_{i}\right]<\infty$. Denotaremos por $T_{i}^{(+)}$os tempos aleatórios de retorno ao zero para as trajetorias de $\nu(t)$ que não cruzam o nível $n$ e por $T^{(-)}$o tempo aleatório de retorno ao zero quando o processo cruza o estado $n$, (falha do sistema). Na Figura (1.1), representamos o tempo de vida do sistema como função dos tempos aleatórios $T_{i}^{(+)}$e $T^{(-)}$ considerando $\nu(0)=0$.

Seja $p_{n}$ a probabilidade de alcançar o estado $n$ entre dois sucessivos retornos ao estado zero, e seja $M_{n}$ a variável aleatória que conta o número de retornos a zero necessários para alcançar o nível $n$. Assim,

$$
P\left(M_{n}=m\right)=\left(1-p_{n}\right)^{m-1} p_{n}
$$

O teorema abaixo foi provado por Keilson (1966) (ver [4]) e analisa as propriedades da distribuição de $\xi=\frac{\tau_{0, n}}{\mu_{0 . n}}$ quando $n \rightarrow \infty$, onde $\mu_{0, n}=\mathbb{E}\left[\tau_{0, n}\right]$. 


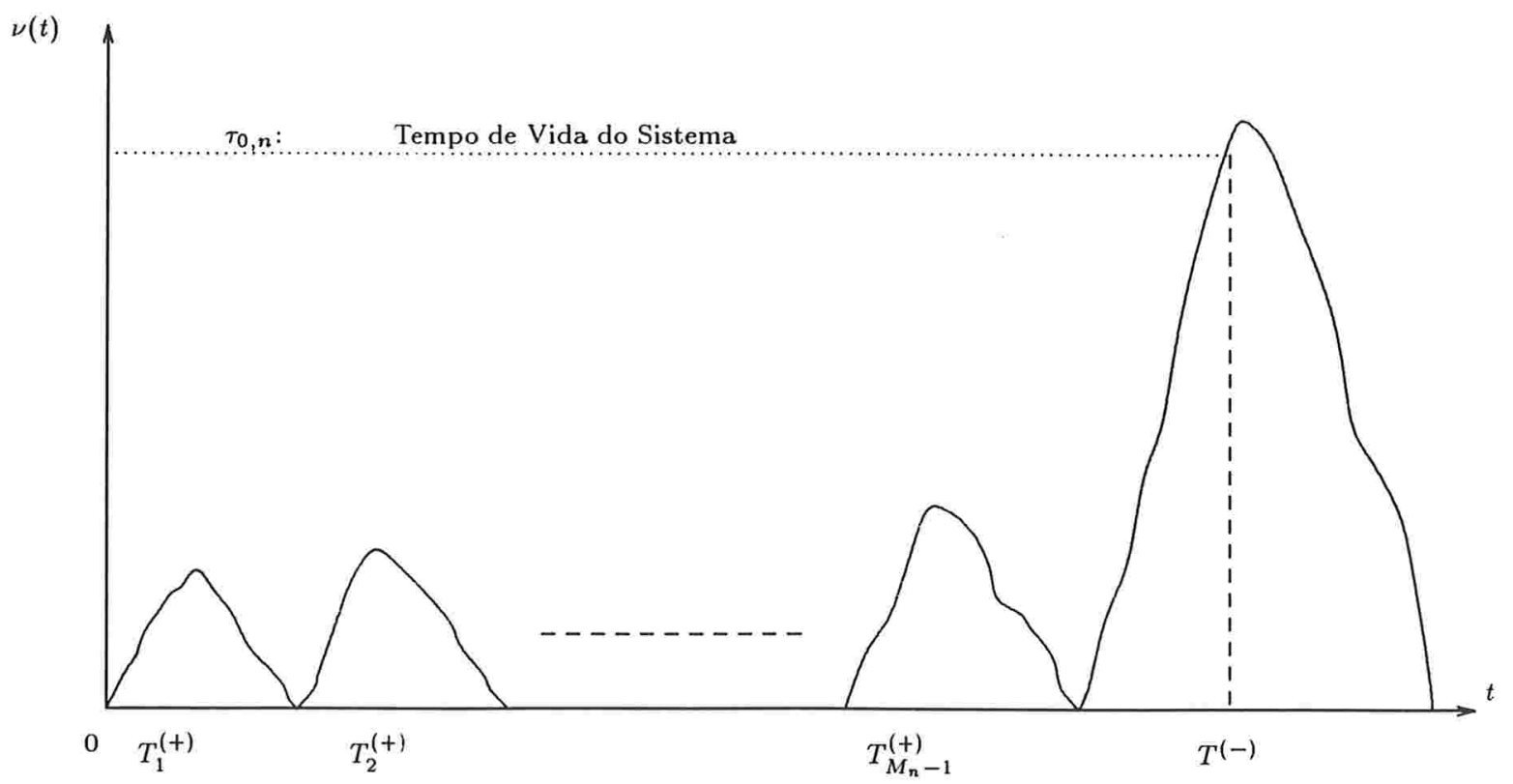

Figura 1.1 Representação do tempo de vida do sistema através de intervalos.

\section{Teorema 1.1.2}

Se $\nu(t)$ é um Processo Ergódico de Nascimento e Morte, então:

$$
P\left(\frac{\tau_{0, n}}{\mu_{0, n}}>t\right) \rightarrow e^{-t}, \text { quando } n \rightarrow \infty
$$

\section{PROVA}

Utilizando a notação acima, observamos que

$$
\begin{array}{rrr}
0 & \leq \tau_{0, n} \leq T^{(-)}, & \text {para } M_{n}=1 \\
T_{1}^{(+)}+\cdots+T_{M_{n}-1}^{(+)} & \leq \tau_{0, n} \leq T_{1}^{(+)}+\cdots+T_{M_{n}-1}^{(+)}+T^{(-)}, & \text {para } M_{n}>1
\end{array}
$$

Se $\varphi^{(+)}(z)$ e $\varphi^{(-)}(z)$ representam as transformadas de Laplace de $T_{i}^{(+)}$e $T^{(-)}$respectivamente, e assumindo $T_{0}^{(+)}=0$ temos de (1.19):

$$
\begin{aligned}
\mathbb{E}\left[e^{-z \tau_{0}, n}\right] & \leq \mathbb{E}\left[e^{-z \sum_{i=0}^{M_{n}-1} T_{i}^{(+)}}\right] \\
& =\sum_{k=0}^{\infty} P\left(M_{n}-1=k\right) \mathbb{E}\left[e^{-z \sum_{i=0}^{M_{n}-1} T_{i}^{(+)}} \mid M_{n}-1=k\right] \\
& =p_{n}+\sum_{k=1}^{\infty} P\left(M_{n}-1=k\right) \mathbb{E}\left[e^{-z \sum_{i=1}^{M_{n}-1} T_{i}^{(+)}} \mid M_{n}-1=k\right]
\end{aligned}
$$




$$
\begin{aligned}
& =p_{n}+\sum_{k=1}^{\infty} P\left(M_{n}-1=k\right) \mathbb{E}\left[e^{-z\left(T_{1}^{(+)}+\cdots+T_{k}^{(+)}\right)}\right] \\
& =p_{n}+\sum_{k=1}^{\infty}\left(1-p_{n}\right)^{k} p_{n}\left[\varphi^{(+)}(z)\right]^{k} \\
& =p_{n}\left[1+\frac{\left(1-p_{n}\right) \varphi^{(+)}(z)}{1-\left(1-p_{n}\right) \varphi^{(+)}(z)}\right]
\end{aligned}
$$

A igualdade acima segue do fato que $M_{n}-1$ é um Tempo de Parada para a sequência $T_{1}, T_{2}, \ldots$ Obtemos então que

$$
\mathbb{E}\left[e^{-z \tau_{0, n}}\right] \leq \frac{p_{n}}{1-\left(1-p_{n}\right) \varphi^{(+)}(z)}
$$

Da mesma forma,

$$
\begin{aligned}
\mathbb{E}\left[e^{-z \tau_{0, n}}\right] & \geq \mathbb{E}\left[e^{-z\left(T_{1}^{(+)}+\cdots+T_{M_{n}-1}^{(+)}+T^{(-)}\right)}\right] \\
& =\sum_{k=0}^{\infty} P\left(M_{n}-1=k\right) \mathbb{E}\left[e^{-z\left(T_{1}^{(+)}+\cdots+T_{M_{n}-1}^{(+)}+T^{(-)}\right)} \mid M_{n}-1=k\right] \\
& =p_{n} \varphi^{(-)}(z)+\sum_{k=1}^{\infty}\left(1-p_{n}\right)^{k} p_{n}\left[\varphi^{(+)}(z)\right]^{k} \varphi^{(-)}(z)
\end{aligned}
$$

de onde

$$
\mathbb{E}\left[e^{-z \tau_{0, n}}\right] \geq \frac{p_{n} \varphi^{(-)}(z)}{1-\left(1-p_{n}\right) \varphi^{(+)}(z)}
$$

Por outro lado, admitindo que $T_{i}^{(+)}$tem função densidade de probabilidade $f_{1}(x)$ e que $T^{(-)}$tem função densidade de probabilidade $f_{2}(x)$ e definindo

$$
\left.\begin{array}{l}
\mu_{0, n}^{(+)}=\mathbb{E}\left[T_{i}^{(+)}\right]=\int_{0}^{\infty} x f_{1}(x) d x \\
\mu_{0, n}^{(-)}=\mathbb{E}\left[T^{(-)}\right]=\int_{0}^{\infty} x f_{2}(x) d x
\end{array}\right\}
$$

provemos que $p_{n} \mu_{0, n}^{(-)} \rightarrow 0$ quando $n \rightarrow \infty$. Se $f(x)$ é a função densidade de probabilidade de $T_{i}$ onde $T_{i}=T_{i}^{(+)}$com probabilidade $\left(1-p_{n}\right)$ e $T_{i}=T^{(-)}$com probabilidade $p_{n}$, temos que

$$
f(x)=\left(1-p_{n}\right) f_{1}(x)+p_{n} f_{2}(x)
$$

Como $\mathbb{E}\left[T_{i}\right]<\infty$,

$$
\lim _{n \rightarrow \infty} \int_{n}^{\infty} x f(x) d x=0
$$


podemos considerar então que, para qualquer $\epsilon>0$ existe $x_{0}>0$ tal que

$$
\int_{x_{0}}^{\infty} x f(x) d x<\epsilon, \quad \text { isto é, } \quad \int_{x_{0}}^{\infty} x\left[\left(1-p_{n}\right) f_{1}(x)+p_{n} f_{2}(x)\right] d x<\epsilon
$$

e portanto,

$$
p_{n} \int_{x_{0}}^{\infty} x f_{2}(x) d x<\epsilon
$$

Por outro lado,

$$
p_{n} \int_{0}^{x_{0}} x f_{2}(x) d x \leq p_{n} x_{0} \int_{0}^{x_{0}} f_{2}(x) d x \leq p_{n} x_{0}
$$

Utilizando as últimas desigualdades e a equação (1.22), temos:

$$
\begin{aligned}
p_{n} \mu_{0, n}^{(-)} & =p_{n} \int_{0}^{x_{0}} x f_{2}(x) d x+p_{n} \int_{x_{0}}^{\infty} x f_{2}(x) d x \\
& \leq p_{n} x_{0}+\epsilon \leq 2 \epsilon \quad \text { quando } \quad p_{n} \leq \frac{\epsilon}{x_{0}}
\end{aligned}
$$

Para mostrar que $p_{n} \rightarrow 0$ quando $n \rightarrow \infty$, observe que $M_{n}-1$ é um tempo de parada e $\mathbb{E}\left(M_{n}\right)=1 / p_{n}$. Assim, pela equação de Wald ([6] pag 31-41),

$$
\mathbb{E}\left[T_{1}^{(+)}+\cdots+T_{M_{n}-1}^{(+)}\right]=\mathbb{E}\left[M_{n}-1\right] \mathbb{E}\left[T_{i}^{(+)}\right]=\left(\frac{1-p_{n}}{p_{n}}\right) \mu_{0, n}^{(+)} .
$$

Concluímos de (1.19) que:

$$
\left(\frac{1-p_{n}}{p_{n}}\right) \mu_{0, n}^{(+)} \leq \mathbb{E}\left[\tau_{0, n}\right] \leq\left(\frac{1-p_{n}}{p_{n}}\right) \mu_{0, n}^{(+)}+\mu_{0, n}^{(-)}
$$

ou

$$
\left(1-p_{n}\right) \mu_{0, n}^{(+)} \leq p_{n} \mathbb{E}\left[\tau_{0, n}\right] \leq\left(1-p_{n}\right) \mu_{0, n}^{(+)}+p_{n} \mu_{0, n}^{(-)}=\mu
$$

Como $\nu(t)$ é um processo ergódico de Nascimento e Morte, $\mathbb{E}\left[\tau_{0, n}\right] \rightarrow \infty$ quando $n \rightarrow \infty$ (ver [1]). Desta maneira,

$$
p_{n} \leq \frac{\mu}{\mathbb{E}\left[\tau_{0, n}\right]} \rightarrow 0 \quad \text { quando } \quad n \rightarrow \infty
$$

Finalmente, seja $\varphi(z)$ a transformada de Laplace da variável aleatória $T_{i}$. De (1.23) e das definições de $\varphi^{(+)}$e $\varphi^{(-)}$, temos:

$$
\mathbb{E}\left[e^{-z T_{i}}\right]=\varphi(z)=\int_{0}^{\infty} e^{-z x} f(x) d x=\left(1-p_{n}\right) \varphi^{(+)}(z)+p_{n} \varphi^{(-)}(z)
$$


se substituímos $z$ por $p_{n} z$ na igualdade acima, teriamos que

$$
\varphi\left(z p_{n}\right)=\int_{0}^{\infty} e^{-z p_{n} x} f(x) d x=\left(1-p_{n}\right) \varphi^{(+)}\left(z p_{n}\right)+p_{n} \varphi^{(-)}\left(z p_{n}\right)
$$

de forma que

$$
\varphi^{(+)}\left(p_{n} z\right)=\frac{\varphi\left(p_{n} z\right)-p_{n} \varphi^{(-)}\left(p_{n} z\right)}{1-p_{n}}
$$

Considerando a inversa do lado direito de (1.20) e substituindo $z$ por $p_{n} z$, temos:

$$
\frac{1-\left(1-p_{n}\right) \varphi^{(+)}\left(p_{n} z\right)}{p_{n}}=z \underbrace{\frac{1-\varphi\left(p_{n} z\right)}{p_{n} z}}_{(*)}+\underbrace{\varphi^{(-)}\left(p_{n} z\right)}_{(* *)}
$$

Para (*) :Foi mostrado acima que quando $n \rightarrow \infty, p_{n} \rightarrow 0$. Observe agora que

$$
-\lim _{p_{n} \rightarrow 0} \frac{\varphi\left(p_{n} z\right)-\varphi(0)}{p_{n} z}=-\lim _{p_{n} \rightarrow 0} \frac{\varphi\left(p_{n} z\right)-1}{p_{n} z}=-\varphi^{\prime}(0)=\mu
$$

Para $(* *)$ Observe que $\varphi^{(-)}\left(p_{n} z\right)=\mathbb{E}\left[e^{-p_{n} z T^{(-)}}\right] \leq 1$ e que, para $y>0,1-e^{-y}=$ $\int_{0}^{y} e^{-x} d x<1$; então:

$$
\begin{aligned}
0 \leq \frac{1-\varphi^{(-)}\left(p_{n} z\right)}{p_{n}} & =\int_{0}^{\infty} \frac{1-e^{-p_{n} z x}}{p_{n} z x} z x f_{2}(x) d x \\
& \leq \int_{0}^{\infty} z x f_{2}(x) d x \\
& =z \mu_{0, n}^{(-)},
\end{aligned}
$$

isto é,

$$
0 \leq 1-\varphi^{(-)}\left(p_{n} z\right) \leq z p_{n} \mu_{0, n}^{(-)}
$$

Em (1.24) e (1.25) foi demonstrado que $p_{n} \mu_{0, n}^{(-)} \rightarrow 0$ quando $n \rightarrow \infty$. Então:

$$
\begin{aligned}
& 0 \leq 1-\varphi^{(-)}\left(p_{n} z\right) \leq z p_{n} \mu_{0, n}^{(-)} \rightarrow 0, \text { quando } n \rightarrow \infty, \quad e \\
& \text { portanto, } \quad \varphi^{(-)}\left(p_{n} z\right) \rightarrow 1, \text { quando } n \rightarrow \infty
\end{aligned}
$$

Voltando para a equação (1.29), observamos que o lado direito tende a $1+\mu z$. De maneira semelhante, considere agora a inversa do lado direito da desigualdade (1.21), 
com $z$ substituído por $p_{n} z$, e a equação (1.28). De forma similar ao procedimento adotado acima, temos que:

$$
\begin{aligned}
\frac{1-\left(1-p_{n}\right) \varphi^{(+)}\left(z p_{n}\right)}{p_{n} \varphi^{(-)}\left(z p_{n}\right)} & =\frac{1-\left(1-p_{n}\right)\left(\frac{\varphi\left(z p_{n}\right)-p_{n} \varphi^{(-)}\left(z p_{n}\right)}{1-p_{n}}\right)}{p_{n} \varphi^{(-)}\left(z p_{n}\right)} \\
& =\frac{1-\varphi\left(z p_{n}\right)}{p_{n} \varphi^{(-)}\left(z p_{n}\right)}+1 \\
& =z\left(\frac{1-\varphi\left(z p_{n}\right)}{z p_{n}}\right)\left(\frac{1}{\varphi^{(-)}\left(z p_{n}\right)}\right)+1 .
\end{aligned}
$$

Como

$$
\lim _{n \rightarrow \infty} \frac{1-\varphi\left(z p_{n}\right)}{z p_{n}}=\mu
$$

concluímos que:

$$
\mathbb{E}\left[e^{-z p_{n} \tau_{0, n}}\right] \rightarrow(1+\mu z)^{-1}
$$

Isto significa que, em distribuição:

$$
\begin{aligned}
P\left(p_{n} \tau_{0, n}>t\right) & \rightarrow e^{-t / \mu} \\
P\left(\frac{p_{n} \tau_{0, n}}{\mu}>t\right) & \rightarrow e^{-t}
\end{aligned}
$$

Em (1.24) achamos que :

$$
\frac{\left(1-p_{n}\right) \mu_{0, n}^{(+)}}{\mu} \leq \frac{p_{n}}{\mu} \mathbb{E}\left[\tau_{0, n}\right] \leq 1
$$

Portanto,

$$
\frac{\mu_{0, n}^{(+)}}{\mu} \rightarrow 1 \quad e \quad p_{n} \mathbb{E}\left[\tau_{0, n}\right] \rightarrow \mu
$$

usando o teorema de Slutsky concluímos a prova do teorema.

\subsubsection{Tempo de Permanência nos estados de funcionamento}

Consideremos agora um sistema em multiestado que passa por vários níveis de degradação e cujos conjuntos de possíveis estados de funcionamento é $\mathcal{E}_{(+)}=\{0,1,2, \ldots, n-1\}$, onde 0 representa o estado de perfeito funcionamento. O conjunto de falha do sistema é 
$\mathcal{E}_{(-)}=\{n, n+1, \ldots\}$. Suponhamos que o sistema possa ser interpretado como um processo ergódico de Nascimento e Morte e que esteja funcionando por um longo período de tempo com probabilidades

$$
P(\nu(t)=k)=p_{k}, \quad k \geq 0,
$$

onde $\nu(t)$ indica o estado do sistema no tempo t.

Consideremos que num instante $t_{0}$ desconhecemos o passado do processo, mas sabemos que $\nu\left(t_{0}\right) \in \mathcal{E}_{(+)}$. Seja $\tau_{e, n}$ o tempo de permanência em $\mathcal{E}_{(+)}$supondo que a distribuição inicial do processo em $\mathcal{E}_{(+)}$é:

$$
p_{i}^{e}=\frac{p_{i}}{\sum_{j \in \mathcal{E}_{(+)}} p_{j}}, \quad i=0,1, \ldots, n-1
$$

Keilson(1979) (ver [4]) prova que $\tau_{e, n} / \mu_{0, n}$ converge em distribuição para uma distribuição exponencial padrão.

\section{Teorema 1.1.3}

Sob as condiçôes do teorema anterior,

$$
P\left(\frac{\tau_{e, n}}{\mu_{0 . n}}>t\right) \rightarrow e^{-t}, \quad \text { quando } \quad n \rightarrow \infty
$$

PROVA

Fixemos um $m>0$ com $m<n$ tal que

$$
\tau_{0, n}=\tau_{0, m}+\tau_{m, n},
$$

onde $\tau_{0, m}=\inf \{t: \nu(t)=m \mid \nu(0)=0\} \quad$ e $\tau_{m, n}=\inf \{t: \nu(t)=n \mid \nu(0)=m\}$. Como $\tau_{0, m}$ e $\tau_{m, n}$ são independentes, temos que:

$$
\begin{aligned}
\varphi_{0, n}\left(\frac{z}{\mu_{0, n}}\right) & =\mathbb{E}\left[e^{-z \tau_{0, n} / \mu_{0, n}}\right]=\mathbb{E}\left[e^{-z\left(\tau_{0, m}+\tau_{m, n}\right) / \mu_{0, n}}\right] \\
& =\mathbb{E}\left[e^{-z \tau_{0, m} / \mu_{0, n}}\right] \mathbb{E}\left[e^{-z \tau_{m, n} / \mu_{0, n}}\right] \\
& =\varphi_{0, m}\left(\frac{z}{\mu_{0, n}}\right) \varphi_{m, n}\left(\frac{z}{\mu_{0, n}}\right)
\end{aligned}
$$


Como $\nu(t)$ é um processo ergódico de Nascimento e Morte, concluímos que $\mu_{0, n} \rightarrow \infty$ quando $n \rightarrow \infty$.

Desta maneira, para um $z>0$ fixado, $\varphi_{0, m}\left(\frac{z}{\mu_{0, n}}\right) \rightarrow \varphi_{0, m}(0)=1$ quando $\quad n \rightarrow \infty$.

No teorema anterior provamos que a transformada de Laplace da variável aleatória $\tau_{0, n} / \mu_{0, n}$ tende a $1 /(1+z)$, que é a transformada de Laplace de uma variável exponencial padrão. Portanto:

$$
\lim _{n \rightarrow \infty} \varphi_{0, n}\left(\frac{z}{\mu_{0, n}}\right)=\lim _{n \rightarrow \infty} \varphi_{m, n}\left(\frac{z}{\mu_{0, n}}\right)=\frac{1}{1+z}
$$

Por definição de $\tau_{e, n}$ temos:

$$
\varphi_{e, n}\left(\frac{z}{\mu_{0, n}}\right)=\mathbb{E}\left[e^{-z \tau_{e, n} / \mu_{0, n}}\right]=\frac{\sum_{m=0}^{n-1} p_{m} \varphi_{m, n}\left(\frac{z}{\mu_{0, n}}\right)}{\sum_{m=0}^{n-1} p_{m}}
$$

De (1.33) e do fato que para $n$ suficientemente grande $\sum_{k=n}^{\infty} p_{k}<\epsilon$, podemos concluir que:

$$
\varphi_{e, n}\left(\frac{z}{\mu_{0, n}}\right) \rightarrow \frac{1}{1+z}
$$

\subsection{Tempos de reparo arbitrariamente distribuídos}

Nesta seção, supomos que os tempos de reparo seguem uma distribuição arbitrária. Para entender a diferença com relação ao processo estudado na seção anterior utilizamos o seguinte exemplo: considere um sistema com uma unidade de reparo e suponha que num certo instante $t, \nu(t)=3$, isto é, o sistema tem uma unidade sendo reparada e duas esperando por atendimento. A transição do processo é observada da seguinte forma: $\mathrm{O}$ tempo de espera para uma nova falha (o que levaria o processo para o estado 4) é exponencial com parâmetro $\lambda$, pois o intervalo entre falhas é exponencial e esta distribuição tem a propriedade de falta de memória. O tempo para completar um reparo (que levaria o processo ao estado 2) não pode ser determinado pois a distribuiçăo em consideração não tem necessariamente a propriedade de falta de memória. A informação de que $\nu(t)=3$ não é suficiente para determinar as probabilidades de transição. 
Assumiremos que o processo $\nu(t)$ se renova em certos instantes de tempo $T_{i}$, isto é, os instantes que $\nu(t)$ assume o valor zero. Os tempos $\xi_{i}=T_{i}-T_{i-1}$ que representam os comprimentos dos intervalos de regeneração, também chamados ciclos de regeneração, são variáveis aleatórias independentes e identicamente distribuídas, com $T_{k}=\sum_{i=1}^{k} \xi_{i}, k \geq 1$. Chamaremos a sequência $\left\{\xi_{i}, i \geq 1\right\}$ processo regenerativo.

\subsubsection{Aparecimento de um evento raro}

Seja $\xi_{i}$ como definida acima e seja $\tau$ o tempo de espera para a ocorrência de um evento $A$. Consideremos $p=P(A)$ a probabilidade de $A$ ocorrer em qualquer período regenerativo. Assim:

$$
\tau=\xi_{1}+\xi_{2}+\cdots+\xi_{N}
$$

onde $N$ é uma variável aleatória com

$$
P(N=n)=(1-p)^{n-1} p, \quad n \geq 1
$$

Para encontrar a distribuição de $\tau$, usaremos a transformada de Laplace.

$$
\begin{aligned}
\mathcal{L}(\tau) & =\mathbb{E}\left(e^{-z \tau}\right)=\mathbb{E}\left[e^{-z\left(\xi_{1}+\cdots+\xi_{N}\right)}\right]=\sum_{k=1}^{\infty} P(N=k) \mathbb{E}\left[e^{-z\left(\xi_{1}+\cdots+\xi_{N}\right)} \mid N=k\right] \\
& =\sum_{k=1}^{\infty}(1-p)^{k-1} p \mathbb{E}\left[e^{-z\left(\xi_{1}+\cdots+\xi_{k}\right)}\right]=\sum_{k=1}^{\infty}(1-p)^{k-1} p[\varphi(z)]^{k}
\end{aligned}
$$

Se os $\xi_{i}$ são exponenciais de parâmetro $1 / \mu$ então,

$$
\varphi(z)=(1+\mu z)^{-1}
$$

e portanto,

$$
\mathcal{L}(\tau)=\frac{p(1+\mu z)^{-1}}{1-(1-p)[1+\mu z]^{-1}}=\left(1+\frac{\mu}{p} z\right)^{-1}
$$

que corresponde à Transformada de Laplace de uma variável aleatória exponencial de parâmetro $p / \mu$, isto é, $\tau \sim \exp (p / \mu)$ 
Se os $\xi_{i}$ têm distribuição arbitrária, o teorema abaixo, provado por Brown (1986) (ver [4]), mostra que, sob algumas condições, a variável aleatória $\tau=\xi_{1}+\cdots+\xi_{N}$ converge para uma variável exponencial.

\section{Teorema 1.2.1}

\section{Sejam:}

1. $\left\{X_{i}, i=1,2, \ldots\right\}$ uma sequência aleatória que satisfaz a Lei Forte dos Grandes Números, isto é:

$$
P\left(\lim _{n \rightarrow \infty} \bar{X}_{n}=\mu\right)=1, \quad 0<\mu<\infty, \quad \text { onde } \bar{X}_{n}=\frac{\sum_{i=1}^{n} X_{i}}{n}
$$

2. A variável aleatória $N$ com distribuição geométrica de parâmetro $p$ e independente da sequência $\left\{X_{i}\right\}$,e

3.

$$
Y=X_{1}+X_{2}+\cdots+X_{N}
$$

Então:

$$
\frac{p}{\mu} Y \stackrel{d}{\rightarrow} \exp (1) \quad \text { quando } p \rightarrow 0
$$

\section{PROVA}

A variável de interesse pode ser expressa na seguinte forma:

$$
\frac{p}{\mu} Y=\frac{p}{\mu} \sum_{i=1}^{N} X_{i}=\frac{p N}{\mu} \frac{\sum_{i=1}^{N} X_{i}}{N}=p N \frac{\bar{X}_{N}}{\mu} .
$$

Contudo,

$$
\begin{aligned}
\mathbb{E}\left[e^{-t p N}\right] & =\sum_{n=1}^{\infty} \mathbb{E}\left[e^{-t p N} \mid N=n\right] P(N=n)=\sum_{n=1}^{\infty}(1-p)^{n-1} p \mathbb{E}\left[e^{-t p N} \mid N=n\right] \\
& =\sum_{n=1}^{\infty}(1-p)^{n-1} p \mathbb{E}\left[e^{-t p n}\right]=\sum_{n=1}^{\infty} \frac{p}{1-p}(1-p)^{n}\left(e^{-t p}\right)^{n} \\
& =\frac{p}{1-p} \frac{(1-p) e^{-t p}}{\left[1-(1-p) e^{-t p}\right]}=\frac{p}{e^{t p}-(1-p)} .
\end{aligned}
$$

Aplicando a regra de L'Hopital ao limite desta transformada de Laplace obtemos:

$$
\lim _{p \rightarrow 0} \mathbb{E}\left[e^{-t p N}\right]=\lim _{p \rightarrow 0}\left(\frac{p}{e^{t p}-(1-p)}\right)=\lim _{p \rightarrow 0}\left(\frac{1}{t e^{t p}+1}\right)=\frac{1}{1+t}
$$


que representa a transformada de Laplace de uma variável aleatória exponencial padrão. Portanto,

$$
p N \stackrel{d}{\rightarrow} \exp (1)
$$

Por hipótese, sabemos que $\frac{\bar{X}_{N}}{\mu}$ converge para 1 com probabilidade 1 . Assim, aplicamos o Teorema de Slutsky e provamos que ${ }_{\mu}^{p} Y$ converge em distribuição a uma variável aleatória exponencial padrão.

Uma importante observação é que as variáveis aleatórias $X_{i}$ não precisam ter o segundo momento finito.

\subsubsection{Processo Regenerativo de duas fases}

Suponhamos que na dinâmica do sistema possamos distinguir dois períodos: períodos livres que correspondem ao estado de perfeito funcionamento do sistema, isto é, $\nu(t)=0$, onde os $\xi_{i}, i=1,2, \ldots$ são tempos aleatórios, independentes e identicamente distribuídos, associados a esses períodos; e os períodos ocupados, correspondentes aos períodos em que ao menos um componente está inoperante, isto é, $\nu(t) \geq 1$. Seja $\eta$ o intervalo de tempo aleatório do período ocupado cujo final corresponde a um ponto de regeneração do processo. A falha do sistema, representada por um evento $A$, pode ocorrer durante qualquer período ocupado, independentemente do passado, com probabilidade $P(A)=p$. Às copias idênticas de $\eta$ denotaremos por $\eta_{i}^{0}$. Denotaremos então por $\eta_{i}^{0}=\eta_{i}^{(+)}$aos comprimentos dos períodos ocupados onde $A$ não ocorre e por $\eta_{i}^{0}=\eta_{n}^{(-)}$ao intervalo onde $A$ aparece. Os instantes de regeneração são os pontos $T_{i}=T_{i-1}+\xi_{i}+\eta_{i}^{0}, T_{0}=0$, $i=1,2, \ldots$ Nesta seção, consideramos que os tempos de vida dos componentes seguem uma distribuição exponencial de parâmetro $\lambda$, isto é, $\xi_{i} \sim \exp (\lambda)$. Assumimos também a hipótese de "reparo rápido": $\mathbb{E}\left[\eta_{i}^{0}\right] \ll \mathbb{E}\left[\xi_{i}\right]$. 


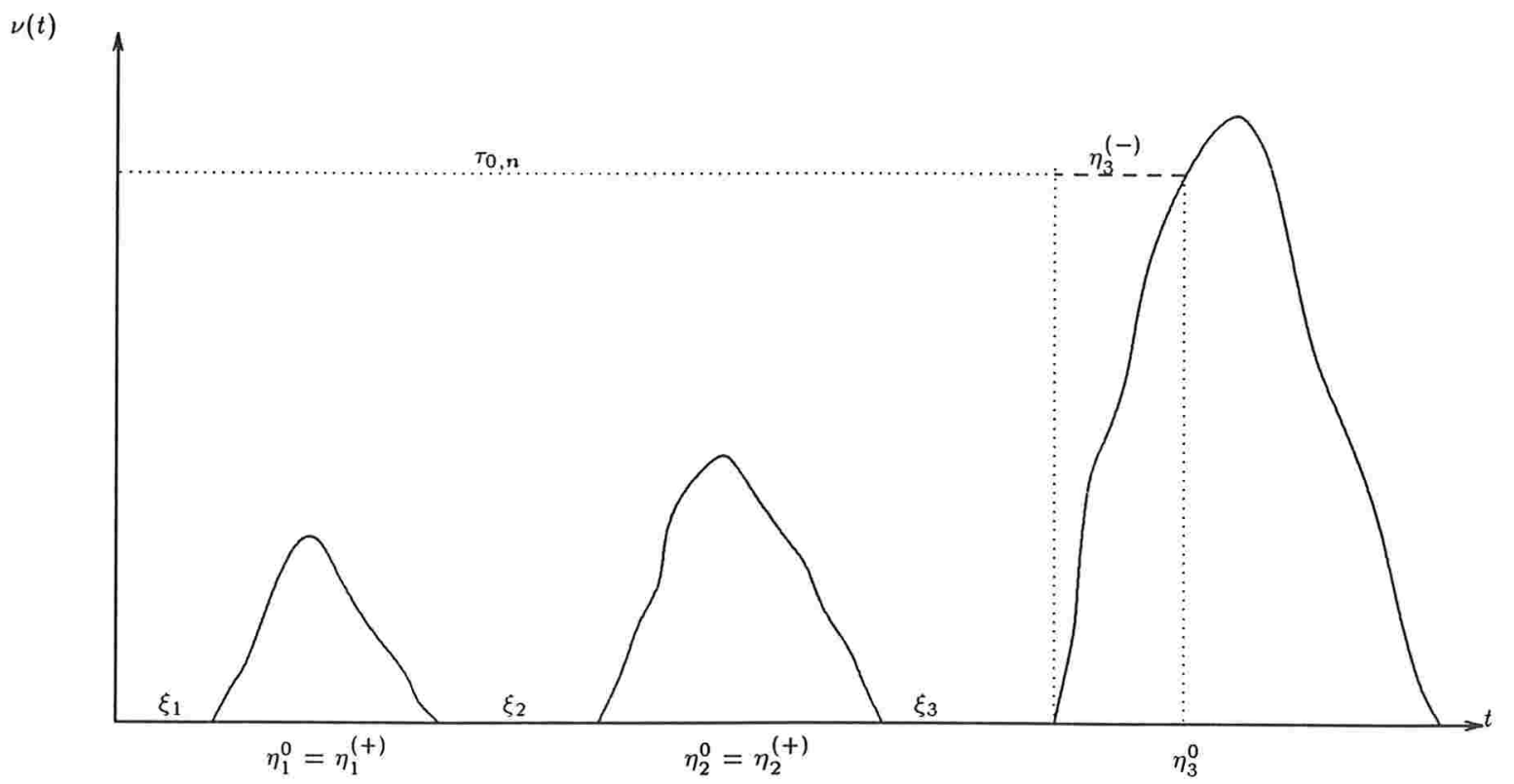

Figura 1.2 Esquema do tempo de vida do sistema considerando períodos livres $e$ ocupados

Se denotamos por $\tau$ o tempo de vida do sistema, temos:

$$
\tau=\xi_{1}+\cdots+\xi_{N}+\eta_{1}^{(+)}+\cdots+\eta_{N-1}^{(+)}+\eta_{N}^{(-)}
$$

onde $N$ tem distribuição geométrica: $P(N=k)=(1-p)^{k-1} p, \quad k \geq 1$.

Seja

$$
\begin{gathered}
\xi=\sum_{i=1}^{N} \xi_{i}, \quad \tilde{\eta}=\sum_{i=1}^{N-1} \eta_{i}^{(+)}+\eta_{N}^{(-)} e \\
\eta_{n}=\eta_{n}^{(+)}\left(1-\chi_{n}\right)+\eta_{n}^{(-)} \chi_{n},
\end{gathered}
$$

onde $\chi_{n}$ é o indicador do evento $A$ :

$$
\chi_{n}= \begin{cases}1 & \text { se } A \text { ocorre no n-ésimo período ocupado, } \\ 0 & \text { caso contrário. }\end{cases}
$$

Assim, $\tau=\xi+\tilde{\eta}$. Sabemos que a soma de um número geometricamente distribuídos de parcelas exponenciais tem uma distribuição exponencial, isto é, $\xi \sim \exp (\lambda p)$. Se o segundo termo $\tilde{\eta}$ pode ser considerado pequeno e desprezível, a distribuição de $\tau$ será 
aproximadamente exponencial. No teorema a seguir, estabelecemos limites para o desvio de $P(\tau>t)$ em relação a $\exp (-\lambda p t)$.

\section{Teorema 1.2.2}

$$
e^{-\lambda p t} \leq P(\tau>t) \leq e^{-\lambda p t}+\lambda \mu^{*} \leq e^{-\lambda p t}+\lambda \mu_{0}
$$

onde $\mu^{*}$ indica o tempo médio de reparo de um servidor.

PROVA:

Claramente, o evento $\{\xi>t\} \subset\{\xi+\tilde{\eta}>t\}$, e concluimos que

$$
P(\tau>t)=P(\xi+\tilde{\eta}>t) \geq P(\xi>t)=e^{-\lambda p t}
$$

O evento $\{\tau>t\}$ pode ser escrito como:

$$
\{\tau>t\}=\{\xi>t\} \quad \text { ou } \quad\{\xi=x, x \in(0, t) \quad \text { e } \quad \tilde{\eta}>t-x\}
$$

Portanto, usando (A.1) temos:

$$
\begin{aligned}
P(\tau>t) & =e^{-\lambda p t}+\int_{0}^{t} \lambda p e^{-\lambda p x} P(\tilde{\eta}>t-x \mid \xi=x) d x \\
& =e^{-\lambda p t}+\int_{0}^{t} \lambda p e^{-\lambda p x} \bar{F}_{x}(t-x) d x \\
& \leq e^{-\lambda p t}+\lambda \mu^{*} \\
& \leq e^{-\lambda p t}+\lambda \mu_{0}
\end{aligned}
$$

e concluimos a prova do teorema.

A justificativa da penúltima desigualdade da prova do teorema, é dada. pelo Lema no Apêndice A

Para exemplificar o uso do teorema acima, consideramos um sistema com componentes reparáveis onde os tempos de vida dos componentes em operação têm distribuições exponenciais. Quando um componente falha, vai para uma unidade de reparo que consta 
de $\mathrm{r}$ servidores independentes. Cada servidor repara um componente falho durante um tempo $\eta$ (supomos aqui que $\eta$ tem uma distribuição arbitrária $G$ ). Definimos $\nu(t)$ como o número de unidades falhadas no sistema no instante $\mathrm{t}$ (este número também inclui as unidades que estão sendo reparadas). O sistema falha quando $\nu(t)$ alcança um certo nível crítico $n$. Este sistema é similar a um denotado em Teoria das Filas por $M / G / r / n-r$, onde $r$ indica o número de servidores ou canais de serviço e $(n-r)$ é a capacidade da fila de espera (sistemas deste tipo serão analisados com mais detalhe no capítulo seguinte).

Considere $\mu=\mathbb{E}[\eta]$ o tempo médio de reparo em um servidor.

\section{Teorema 1.2.3}

Se $\quad \lambda \mu \rightarrow 0, \quad$ então

$$
P(\lambda p \tau>t) \rightarrow e^{-t}
$$

\section{PROVA}

Mostraremos que a condição do teorema implica em $\lambda \mu_{0} \rightarrow 0$, onde $\mu_{0}=\mathbb{E}\left[\eta_{0}\right]$ indica o tamanho médio do período ocupado para o sistema $M / G / r / n-r$. Se substituímos este sistema por um sistema $M / G / 1$, observamos que o período ocupado para o primeiro sistema é, em média, menor que o período ocupado para o segundo. Também, pela Teoria das Filas (Ver [5],pags 84 e 248)), temos que o tamanho médio do período ocupado para o sistema $M / G / 1$ é igual ao do $M / M / 1$, a saber

$$
\widehat{\mu}_{0}=\frac{\mu}{1-\lambda \mu} .
$$

Assim,

$$
\mu_{0} \leq \widehat{\mu}_{0}=\frac{\mu}{1-\lambda \mu}
$$

e, portanto,

$$
\lambda \mu_{0} \leq \lambda \hat{\mu}_{0}=\lambda \frac{\mu}{1-\lambda \mu} \rightarrow 0
$$

Logo o teorema 1.2.3 segue do teorema 1.2.2. 


\section{Capítulo 2}

\section{Confiabilidade de sistemas com}

\section{componentes reparáveis e}

\section{distribuições arbitrárias}

\subsection{Definições e Notações}

Neste capítulo procuramos uma forma geral para o problema tratado no capítulo anterior. Consideramos que tanto as distribuições dos tempos de vida como dos tempos de reparo são arbitrários. Abordaremos o problema utilizando a linguagem de Teoria das Filas. A Figura 2.1 nos permite uma noção gráfica do problema.

Considere uma fila $(r, m-r)$, isto é, há $r$ dispositivos de serviço e a capacidade da fila de espera é de $m-r$. 


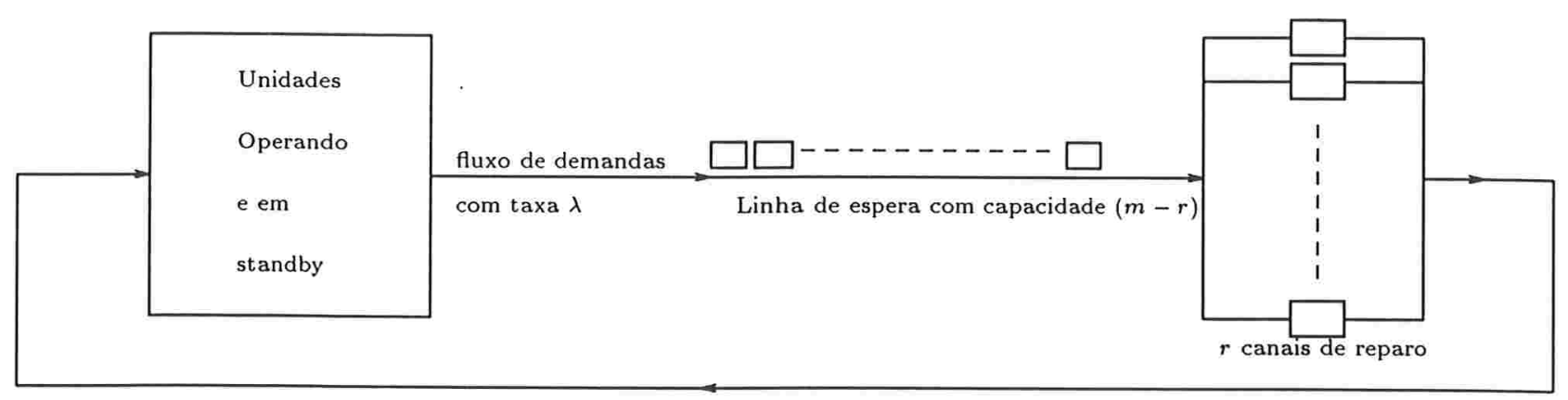

Retorno das unidades reparadas para servic/standby

Figura 2.1 Esquema representando uma fila

O sistema falha no instante em que chega uma demanda a procura de serviço e encontra todos os dispositivos de reparo e a fila de espera ocupados, o que chamaremos de instante da perda de demanda. A hipótese de "reparo rápido" é colocada da seguinte maneira: o tempo médio de reparo de um componente é muito menor que seu tempo médio de falha. Adequando a descrição da operação do sistema para a linguagem de Teoria das Filas, interpretamos as falhas dos componentes como demandas a procura de atendimento e usamos indistintamente canais de serviço ou unidades de reparo para os dispositivos que recuperam os componentes ao seu estado original. O problema resume-se no seguinte: há um fluxo de demandas constituíndo um processo de renovação para um sistema de filas com $r$ canais de serviço e $m-r$ lugares de espera e o nosso objetivo é achar a distribuição do instante da primeira perda de demanda. Os instantes em que as demandas ocorrem são denotados por:

$$
t_{0}=0<t_{1}<t_{2} \ldots<t_{n}<\ldots
$$

e este fluxo é considerado regenerativo.

Os intervalos entre demandas estão representados pelas variáveis aleatórias

$$
\xi_{n}=t_{n}-t_{n-1}
$$

e são independentes e identicamente distribuídos de acordo com uma função de distribuição de probabilidade

$$
F(x)=P\left(\xi_{n}<x\right), e \quad \bar{F}(x)=1-F(x) .
$$


O tempo de serviço de uma unidade é denotada por $\eta_{n}$ e comporta-se de acordo com função de distribuição

$$
G(x)=P\left(\eta_{n} \leq x\right), \quad \bar{G}(x)=1-G(x)
$$

Consideramos que os $\xi_{i}$ e $\eta_{j}(i, j=1,2, \ldots)$ são independentes. O tempo para a "perda de uma demanda" é denotado por $\tau$ e desejamos encontrar a classe de distribuições limite para as quais $\tau$ pode convergir sob certas condições.

Denotamos por $\nu=\left\{\nu_{t}\right\}_{t \geq 0}$ o processo aleatório que registra o estado do sistema em função do tempo. $\nu$ é um processo regenerativo cujos pontos de regeneração são alguns dos instantes $t_{n}$ denotados por

$$
T_{0}=0<T_{1}=t_{k_{1}}<T_{2}=t_{k_{2}}<\ldots<T_{n}=t_{k_{n}}<\ldots
$$

os quais correspondem a retornos ao estado zero.

Assumimos que o fluxo de demandas é independente do estado do sistema. Isto significa que, para $n$ arbitrário, o fluxo posterior a $t_{n}$ independe do comportamento de $\nu(t)$ anterior a $t_{n}$. Note que os pontos de regeneração do processo são os instantes $t_{n^{-}}$, antes dos quais não há demandas no sistema (instantes imediatamente anteriores à chegada de uma demanda).

Seja $\zeta=\min \left\{\tau, T_{1}\right\}$, isto é, $\zeta$ é o tempo do primeiro período de regeneração de $\nu(t)$, isto é, $\zeta=T_{1}$ se nenhuma perda de demanda ocorre neste período ou $\zeta=\tau$, caso contrário.

Como $\xi_{n}=t_{n}-t_{n-1}$, temos que

$$
\zeta=\xi_{1}+\xi_{2}+\cdots+\xi_{N}=t_{N}
$$

Portanto, o comportamento assintótico do instante da primeira perda de uma demanda $\tau$ depende do comportamento da variável aleatória a valores inteiros $N$.

Sejam os eventos:

$$
A_{n}^{+}=\left\{N=n, \tau>T_{1}\right\}, \quad A_{n}^{-}=\left\{N=n, \tau \leq T_{1}\right\},
$$




$$
\begin{gathered}
A^{+}=\cup_{n=1}^{\infty} A_{n}^{+}=\left\{\tau>T_{1}\right\}, \quad A^{-}=\cup_{n=1}^{\infty} A_{n}^{-}=\left\{\tau \leq T_{1}\right\}, \\
A_{n}=A_{n}^{+} \cup A_{n}^{-}=\{N=n\}, \\
B_{n}^{+}=\left\{N \geq n, \tau>T_{1}\right\}, \quad B_{n}^{-}=\left\{N \geq n, \tau \leq T_{1}\right\}, \\
B_{n}=B_{n}^{+} \cup B_{n}^{-}=\{N \geq n\},
\end{gathered}
$$

e $I_{\left\{A_{n}^{+}\right\}}, I_{\left\{A_{n}^{-}\right\}}, I_{\left\{A^{+}\right\}}, I_{\left\{A^{-}\right\}}, I_{\left\{A_{n}\right\}}, I_{\left\{B_{n}^{+}\right\}}, I_{\left\{B_{n}^{-}\right\}}, I_{\left\{B_{n}\right\}}$, as respectivas funções indicadoras. $\mathrm{O}$ evento $A^{-}$indica a perda de uma demanda no primeiro periodo de regeneração de $\nu(t)$. Denotamos a probabilidade deste evento por $q$, isto é,

$$
P\left(A^{-}\right)=P\left(\tau \leq T_{1}\right)=q
$$

A Transformada de Laplace da variável aleatória $\zeta$ é definida por $\varphi(z)=\mathbb{E}\left[e^{-z \zeta}\right]$ e será representada por:

$$
\varphi(z)=\varphi_{-}(z)+\varphi_{+}(z)
$$

onde

$$
\begin{gathered}
\varphi_{+}(z)=\mathbb{E}\left[e^{-z \zeta} I_{\left\{A^{+}\right\}}\right]=\mathbb{E}\left[e^{-z \zeta} I_{\left\{\tau>T_{1}\right\}}\right] \quad e \\
\varphi_{-}(z)=\mathbb{E}\left[e^{-z \zeta} I_{\left\{A^{-}\right\}}\right]=\mathbb{E}\left[e^{-z \zeta} I_{\left\{\tau \leq T_{1}\right\}}\right] .
\end{gathered}
$$

Se denotamos a Transformada de Laplace da variável aleatória $\tau$ por $a(z)=\mathbb{E}\left[e^{-z \tau}\right]$ e consideramos o "argumento da renovação":

dado que $A^{-}$ocorre, $\tau=\zeta$,

dado que $A^{-}$não ocorre, $\tau=T_{1}+\tau^{\prime}$, onde $\tau^{\prime}$ é independente de $T_{1}$ e identicamente distribuído a $\tau$, então

$$
\begin{aligned}
a(z) & =\mathbb{E}\left[e^{-z \tau}\right]=\mathbb{E}\left[e^{-z \tau} I_{\left\{\tau \leq T_{1}\right\}}\right]+\mathbb{E}\left[e^{-z \tau} I_{\left\{\tau>T_{1}\right\}}\right] \\
& =\mathbb{E}\left[e^{-z \zeta} I_{\left\{\tau \leq T_{1}\right\}}\right]+\mathbb{E}\left[e^{-z\left(T_{1}+\tau^{\prime}\right)} I_{\left\{\tau>T_{1}\right\}}\right] \\
& =\mathbb{E}\left[e^{-z \zeta} I_{\left\{\tau \leq T_{1}\right\}}\right]+\mathbb{E}\left[e^{-z \zeta} I_{\left\{\tau>T_{1}\right\}}\right] \mathbb{E}\left[e^{-z \tau^{\prime}}\right] \\
& =\varphi_{-}(z)+\varphi_{+}(z) a(z) .
\end{aligned}
$$


Portanto,

$$
a(z)=\mathbb{E}\left[e^{-z \tau}\right]=\frac{\varphi_{-}(z)}{1-\varphi_{+}(z)}=\frac{\varphi_{-}(z)}{\left.1-\varphi_{(}\right)+\varphi_{-}(z)}
$$

\subsection{Teorema Principal}

O lema a seguir fornece um limite para a Transformada de Laplace de $\tau$, e encontra-se demonstrado em [7].

\section{Lema 2.2 .1}

Sejam $q>0$,

$$
\begin{array}{r}
f(z)=\mathbb{E}\left[e^{-z \xi}\right]=\int_{0}^{\infty} e^{-z x} d F(x), \\
\beta=\mathbb{E}[N]-1, \quad e \\
\delta=\sup _{z>0} \frac{\mathbb{E}\left[\left(1-e^{-z \xi_{1}}\right) I_{\left\{\tau \leq T_{1}\right\}}\right]}{\mathbb{E}\left[1-e^{-z \xi_{1}}\right]} .
\end{array}
$$

Então, para $z \geq 0$ arbitrário, temos:

$$
\left|\mathbb{E}\left[e^{-z \tau}\right]-[V(z)+1]^{-1}\right| \leq \beta+\delta,
$$

onde

$$
V(z)=\frac{1-f(z)}{q}
$$

PROVA:

A partir de (2.1) temos que

$$
\begin{aligned}
\mathbb{E}\left[e^{-z \tau}\right] & =\frac{\varphi_{-}(z)}{1-\varphi(z)+\varphi_{-}(z)}=\frac{\varphi_{-}(z)}{q}\left[\frac{q}{1-\varphi(z)+\varphi_{-}(z)}\right] \\
& =\frac{\varphi_{-}(z)}{q}\left[\frac{1-\varphi(z)+\varphi_{-}(z)}{q}\right]^{-1}
\end{aligned}
$$

Denotando $W(z)=\frac{\varphi-(z)}{q}$, podemos escrever:

$$
\mathbb{E}\left[e^{-z \tau}\right]=W(z)\left[\frac{1-\varphi(z)}{q}+W(z)\right]^{-1} .
$$


Contudo,

$$
1-\varphi(z)=\mathbb{E}\left[1-e^{-z \zeta}\right]=\sum_{n=1}^{\infty} \mathbb{E}\left[1-e^{-z t_{n}}\right] I_{\{N=n\}}
$$

Como

$$
I_{\{N=n\}}=I_{\{N \geq n\}}-I_{\{N \geq n+1\}}
$$

e $I_{\{N \geq 1\}} \equiv 1$, obtemos:

$$
\begin{aligned}
1-\varphi(z) & =1-\sum_{n=1}^{\infty} \mathbb{E}\left[e^{-z t_{n}} I_{\{N \geq n\}}\right]+\sum_{n=1}^{\infty} \mathbb{E}\left[e^{-z t_{n}} I_{\{N \geq n+1\}}\right] \\
& =\mathbb{E}\left[e^{-z t_{0}} I_{\{N \geq 1\}}\right]+\sum_{n=1}^{\infty} \mathbb{E}\left[e^{-z t_{n}} I_{\{N \geq n+1\}}\right]-\sum_{n=1}^{\infty} \mathbb{E}\left[e^{-z t_{n}} I_{\{N \geq n\}}\right] \\
& =\sum_{n=1}^{\infty} \mathbb{E}\left[e^{-z t_{n-1}} I_{\{N \geq n\}}\right]-\sum_{n=1}^{\infty} \mathbb{E}\left[e^{-z t_{n}} I_{\{N \geq n\}}\right] \\
& =\sum_{n=1}^{\infty} \mathbb{E}\left[\left(e^{-z t_{n-1}}-e^{-z t_{n-1}-z \xi_{n}}\right) I_{\{N \geq n\}}\right] \\
& =\mathbb{E}\left[e^{-z t_{0}}\left(1-e^{-z \xi}\right) I_{\{N \geq 1\}}\right]+\sum_{n=2}^{\infty} \mathbb{E}\left[e^{-z t_{n-1}}\left(1-e^{-z \xi_{n}}\right) I_{\{N \geq n\}}\right] \\
& =\mathbb{E}\left[1-e^{-z \xi}\right]+\sum_{n=2}^{\infty} \mathbb{E}\left[e^{-z t_{n-1}}\left(1-e^{-z \xi_{n}}\right) I_{\{N \geq n\}}\right]
\end{aligned}
$$

com $t_{0}=0$ nas expressões acima.

Uma vez que o somando do lado direito é positivo, concluímos que

$$
1-\varphi(z) \geq 1-f(z)
$$

Como as quantidades $\xi_{n}$ e $I_{\{N \geq n\}}$ são independentes e para todo $t_{n}$ positivo, $e^{-z t_{n}} \leq 1$ para todo $n \geq 1$ concluímos:

$$
\begin{aligned}
1-\varphi(z) & \leq \sum_{n=1}^{\infty} \mathbb{E}\left[\left(1-e^{-z \xi_{n}}\right) I_{\{N \geq n\}}\right] \\
& =\sum_{n=1}^{\infty} \mathbb{E}\left[1-e^{-z \xi_{n}}\right] \mathbb{E}\left[I_{\{N \geq n\}}\right] \\
& =\mathbb{E}\left[1-e^{-z \xi}\right] \sum_{n=1}^{\infty} P(N \geq n) \\
& =[1-f(z)] \mathbb{E}[N]=[1-f(z)](\beta+1) .
\end{aligned}
$$


Portanto, de (2.2)

$$
1-\varphi(z)=[1-f(z)](1+\theta \beta), \text { para algum } 0 \leq \theta \leq 1,
$$

Por outro lado,

$$
\begin{gathered}
P\left(\tau \leq T_{1}\right)=q=\mathbb{E}\left[I_{\left\{\tau \leq T_{1}\right\}}\right]=\mathbb{E}\left[I_{\left\{N \geq 1, \tau \leq T_{1}\right\}}\right], e \\
\varphi_{-}(z)=\mathbb{E}\left[e^{-z \zeta} I_{\left\{\tau \leq T_{1}\right\}}\right]=\sum_{n=1}^{\infty} \mathbb{E}\left[e^{-z t_{n}} I_{\left\{N=n, \tau \leq T_{1}\right\}}\right] .
\end{gathered}
$$

Segue-se que:

$$
\begin{aligned}
q-\varphi_{-}(z)= & \mathbb{E}\left[e^{-z t_{0}} I_{\left\{N \geq 1, \tau \leq T_{1}\right\}}\right]-\sum_{n=1}^{\infty} \mathbb{E}\left[e^{-z t_{n}}\left(I_{\left\{N \geq n, \tau \leq T_{1}\right\}}-I_{\left\{N \geq n+1, \tau \leq T_{1}\right\}}\right)\right] \\
= & \sum_{n=1}^{\infty} \mathbb{E}\left[e^{-z t_{n-1}}\left(1-e^{-z \xi_{n}}\right) I_{\left\{N \geq n, \tau \leq T_{1}\right\}}\right] \\
\leq & \sum_{n=1}^{\infty} \mathbb{E}\left[\left(1-e^{-z \xi_{n}}\right) I_{\left\{N \geq n, \tau \leq T_{1}\right\}}\right] \\
\leq & \mathbb{E}\left[\left(1-e^{-z \xi_{1}}\right) I_{\left\{\tau \leq T_{1}\right\}}\right]+\sum_{n=2}^{\infty} \mathbb{E}\left[\left(1-e^{-z \xi_{n}}\right) I_{\{N \geq n\}}\right] \\
= & \frac{\mathbb{E}\left[\left(1-e^{-z \xi_{1}}\right) I_{\left\{\tau \leq T_{1}\right\}}\right] \mathbb{E}\left[1-e^{-z \xi_{1}}\right]}{\mathbb{E}\left[1-e^{-z \xi_{1}}\right]}+\sum_{n=1}^{\infty} \mathbb{E}\left[\left(1-e^{-z \xi_{n}}\right) I_{\{N \geq n\}}\right]- \\
& -\mathbb{E}\left[\left(1-e^{-z \xi_{1}}\right) I_{\{N \geq 1\}}\right] \\
\leq & \delta \mathbb{E}\left[1-e^{-z \xi_{1}}\right]+\mathbb{E}\left[1-e^{-z \xi}\right] \sum_{n=1}^{\infty} P(N \geq n)-\mathbb{E}\left[1-e^{-z \xi_{1}}\right] \\
= & \delta(1-f(z))+(1-f(z)) \mathbb{E}[N]-(1-f(z)) \\
= & (1-f(z))(\delta+\mathbb{E}[N]-1) .
\end{aligned}
$$

Concluímos por (2.2) que:

$$
q-\varphi_{-}(z) \leq(1-f(z))(\delta+\beta) .
$$

Então,

$$
\begin{aligned}
& \frac{\varphi_{-}(z)}{q} \geq 1-\frac{(1-f(z))}{q}(\delta+\beta), e \\
& \frac{\varphi_{-}(z)}{q}=1-\theta^{\prime} \frac{(1-f(z))}{q}(\delta+\beta), \text { para algum } 0 \leq \theta^{\prime} \leq 1
\end{aligned}
$$


Portanto,

$$
W(z)=1-\theta^{\prime} V(z)(\delta+\beta), \quad \text { onde }
$$

$\theta^{\prime} \in[0,1], \delta>0$ e $\beta>0$ arbitrários. Então:

$$
\begin{aligned}
\left|\mathbb{E}\left(e^{-z \tau}\right)-[V(z)+1]^{-1}\right|= & \left|W(z)\left[\frac{1-\varphi(z)}{q}+W(z)\right]^{-1}-[V(z)+1]^{-1}\right| \\
= & \mid\left(1-\theta^{\prime} V(z)(\delta+\beta)\right)\left[V(z)(1+\theta \beta)+\left(1-\theta^{\prime} V(z)(\delta+\beta)\right)\right]^{-1}- \\
& -[V(z)+1]^{-1} \mid \\
= & \left|\frac{1-\theta^{\prime} V(z)(\delta+\beta)}{V(z)(1+\theta \beta)+1-\theta^{\prime} V(z)(\delta+\beta)}-\frac{1}{V(z)+1}\right| \\
= & \left|\frac{\theta^{\prime}[V(z)]^{2}(\delta+\beta)+V(z) \theta \beta}{(V(z)+1)\left[V(z)(1+\theta \beta)+1-\theta^{\prime} V(z)(\delta+\beta)\right]}\right| \\
= & \left|\frac{V(z)\left[\theta^{\prime} V(z)(\delta+\beta)+\theta \beta\right]}{[V(z)+1]} \frac{1}{\left[V(z)(1+\theta \beta)+1-\theta^{\prime} V(z)(\delta+\beta)\right]}\right|
\end{aligned}
$$

Observe que

$$
\begin{aligned}
V(z)(1+\theta \beta)+1-\theta^{\prime} V(z)(\delta+\beta) & =\frac{1-\varphi(z)}{q}+\frac{\varphi_{-}(z)}{q} \\
& \geq \frac{1-\varphi(z)}{q}=V(z)+V(z) \theta \beta \geq V(z) \geq 0
\end{aligned}
$$

e, portanto,

$$
\begin{aligned}
\left|\mathbb{E}\left[e^{-z \tau}\right]-[V(z)+1]^{-1}\right| & \leq\left|\frac{\left[\theta^{\prime} V(z)(\delta+\beta)+\theta \beta\right]}{[V(z)+1]}\right| \\
& \leq \frac{V(z)(\delta+\beta)+\beta+\delta}{V(z)+1} \\
& =\delta+\beta
\end{aligned}
$$

Logo, o lema está provado.

Nosso objetivo é achar uma família de distribuições limite para a qual $\tau$ pode convergir e as condições para que esta convergência ocorra. No que segue, apresentamos condições para a convergência da Transformada de Laplace da variável aleatória $\tau$ para uma classe ampla de distribuições. 


\section{Teorema 2.2.1}

Se na notação do Lema 2.2.1,

$$
q>0, \quad q \rightarrow 0, \quad \beta \rightarrow 0, \quad \delta \rightarrow 0,
$$

e se para algum fator de normalização $\gamma, \gamma \tau$ converge para uma variável aleatória com distribuição própria. então

$$
\lim _{q \rightarrow 0} \mathbb{E}\left[e^{-z \gamma \tau}\right]=[1+w(z)]^{-1}
$$

onde

$$
w(z)=\int_{0}^{\infty} \frac{1-e^{-z x}}{x} d P(x),
$$

e $P(x)$ é uma função monótona crescente que satisfaz as condições

$$
P(0)=0, \quad \int_{1}^{\infty} \frac{d P(x)}{x}<\infty
$$

Uma condição necessária e suficiente para a convergência em (2.4) é que

$$
\lim _{q \rightarrow 0} \int_{0}^{x} \frac{t}{q} d F\left(\frac{t}{\gamma}\right)=P(x), x>0 .
$$

\section{PROVA}

Desde que $\beta \rightarrow 0$ e $\delta \rightarrow 0$, por (2.3), segue-se que

$$
\begin{aligned}
\lim _{q \rightarrow 0} \mathbb{E}\left[e^{-z \gamma \tau}\right] & =\lim _{q \rightarrow 0}[V(z \gamma)+1]^{-1} \\
& =\lim _{q \rightarrow 0}\left[\frac{1-f(z \gamma)}{q}+1\right]^{-1} .
\end{aligned}
$$

Como $q \rightarrow 0$, temos: $f(z \gamma) \rightarrow 1$. nas vizinhanças de 1 , temos que pela expansão de Taylor,

$$
\begin{gathered}
\frac{1-f(z \gamma)}{q} \sim-\frac{1}{q} \ln f(z \gamma) \quad e \\
\lim _{q \rightarrow 0} \frac{1-f(z \gamma)}{q}=\lim _{n \rightarrow \infty}-\ln f(z \gamma)^{n} .
\end{gathered}
$$


Concluímos que $\tau$ é uma variável aleatória não negativa infinitamente divisível, e portanto,

$$
\lim _{q \rightarrow 0} \frac{1-f(z \gamma)}{q}=-\ln \chi(z),
$$

onde $\chi(z)$ é a Transformada de Laplace de uma variável aleatória não negativa com distribuição infinitamente divisível. Como por hipótese

$$
\int_{1}^{\infty} \frac{d P(x)}{x}<\infty
$$

usamos o teorema 2 , pag. 426 de [3] e concluímos que $\chi(z)$ tem a forma:

$$
\chi(z)=e^{-w(z)}
$$

Consequentemente,

$$
\begin{aligned}
\lim _{q \rightarrow 0} \mathbb{E}\left[e^{-z \gamma \tau}\right] & =\lim _{q \rightarrow 0}\left[\frac{1-f(z \gamma)}{q}+1\right]^{-1} \\
& =[1-\ln \chi(z)]^{-1} \\
& =\left[1-\ln e^{-w(z)}\right]^{-1}=[1+w(z)]^{-1}
\end{aligned}
$$

Mostramos que

$$
\frac{1-f(z \gamma)}{q} \rightarrow w(z)
$$

isto é,

$$
\begin{aligned}
w(z) & =\lim _{q \rightarrow 0} \frac{1}{q}\left\{1-\int_{0}^{\infty} e^{-z \gamma y} d F(y)\right\}, \quad \text { se } \gamma y=x \\
& =\lim _{q \rightarrow 0} \frac{1}{q}\left\{\int_{0}^{\infty}\left(1-e^{-z x}\right) d F\left(\frac{x}{\gamma}\right)\right\} \\
& =\lim _{q \rightarrow 0}\left\{\int_{0}^{\infty} \frac{1-e^{-z x}}{x} \frac{x}{q} d F\left(\frac{x}{\gamma}\right)\right\}
\end{aligned}
$$

de maneira que para todo $m>0$, existe $\delta(m)>0$ tal que, se $|q|<\delta(m)$ então,

$$
\left|\int_{0}^{\infty}\left(\frac{1-e^{-z x}}{x}\right) \frac{x}{q} d F\left(\frac{x}{\gamma}\right)-w(z)\right|<\frac{1}{m}
$$


Provaremos agora que $w(z)$ tem a forma

$$
w(z)=\int_{0}^{\infty} \frac{1-e^{-z x}}{x} d P(x)
$$

se e somente se, para arbitrário $x>0$,

$$
\lim _{q \rightarrow 0} \int_{0}^{x} \frac{t}{q} d F\left(\frac{t}{\gamma}\right)=P(x)
$$

ou seja,

para todo $n>0$, existe $\delta(n)>0$ tal que se $|q|<\delta(n)$ temos

$$
\left|\int_{0}^{x} \frac{t}{q} d F\left(\frac{t}{\gamma}\right)-P(x)\right|<\frac{1}{n} .
$$

Para mostrar que a condição é suficiente, utilizamos a desigualdade modular:

$$
\begin{aligned}
\left|w(z)-\int_{0}^{\infty} \frac{1-e^{-z x}}{x} d P(x)\right| & \leq\left|w(z)-\int_{0}^{\infty} \frac{1-e^{-z x}}{x} \frac{x}{q} d F\left(\frac{x}{\gamma}\right)\right|+ \\
& +\left|\int_{0}^{\infty}\left(\frac{1-e^{-z x}}{x}\right)\left(\frac{x}{q} d F\left(\frac{x}{\gamma}\right)-d P(x)\right)\right| .
\end{aligned}
$$

Como

$$
\frac{1-e^{-z x}}{x}=\frac{1}{x} \int_{0}^{x} z e^{-z s} d s \leq z
$$

utilizamos (2.8) para concluir que

$$
\left|w(z)-\int_{0}^{\infty} \frac{1-e^{-z x}}{x} d P(x)\right| \leq \frac{1}{m}+z \frac{1}{n} \rightarrow 0 .
$$

Para provar a condição necessária observamos que

$$
\begin{aligned}
\left|\int_{0}^{\infty}\left(\frac{1-e^{-z x}}{x}\right)\left(d P(x)-\frac{x}{q} d F\left(\frac{x}{\gamma}\right)\right)\right| & \leq\left|w(z)-\int_{0}^{\infty} \frac{1-e^{-z x}}{x} d P(x)\right|+ \\
& +\left|w(z)-\int_{0}^{\infty} \frac{1-e^{-z x}}{x} \frac{x}{q} d F\left(\frac{x}{\gamma}\right)\right|
\end{aligned}
$$

de onde concluímos a prova do teorema. 
OBSERVAÇÃO 2.1 Em particular, para a convergência para uma distribuição exponencial, é necessario e suficiente que:

$$
\lim _{q \rightarrow 0} \int_{0}^{x} \frac{t}{q} d F\left(\frac{t}{\gamma}\right)=a=\text { constante }>0, x>0 .
$$

Para provar esta convergência, observe primeiro na definição da função de distribuição de $\xi$

$$
F(x)=P(\xi<x)
$$

isto implica continuidade à esquerda, e como as condições do teorema estabelecem $P(0)=$ 0 e $P(x)=a$, para todo $x>0$, (isto é, o salto no ponto 0 é de tamanho $a$ ), podemos usar uma das propriedades da integral de Riemann-Stieltjes no intervalo $[0, \infty)$, isto é, se $\phi(x)$ é uma função continua num intervalo $[b, c)$, então, a integral de Riemann-Stieljes ignora um eventual salto em $c$ e só leva em consideração um eventual salto no ponto $b$.

Considere $\phi(x)=e^{-z x}$, esta função é continua no intervalo $[0, \infty)$, assím, a integral de Riemann-Stieltjes de $\phi(x)$ em $[0, \infty)$ ponderada por $P(x)$ será:

$$
\int_{0}^{\infty} \phi(x) d P(x)=\int_{0}^{\infty} e^{-z x} d P(x)=a \phi(0)=a .
$$

Nas vizinhanças de zero, temos que

$$
\begin{gathered}
\frac{d}{d x}\left(e^{-z x}\right)=\lim _{x \rightarrow 0} \frac{e^{-z x}-1}{x-0}=-\lim _{x \rightarrow 0} \frac{1-e^{-z x}}{x}, \quad e \\
\frac{d e^{-z x}}{d x}=-z e^{-z x}
\end{gathered}
$$

Portanto, de (2.11) e (2.12), temos que

$$
\lim _{x \rightarrow 0} \int_{0}^{\infty} \frac{1-e^{-z x}}{x} d P(x)=z \int_{0}^{\infty} e^{-z x} d P(x)=z a=c t e
$$

OBSERVAÇÃO 2.2 Para o sistema de serviço $(r, m-r)$, onde $m>1$, temos que

$$
I_{A_{1}^{-}}=I_{\left\{N=1, \tau \leq T_{1}\right\}} \equiv 0
$$


isto é, não haverá perda de demanda no primeiro passo. Então,

$$
\begin{aligned}
q=P\left(\tau \leq T_{1}\right)=\mathbb{E}\left[I_{\left\{A^{-}\right\}}\right] & =\sum_{n=1}^{\infty} \mathbb{E}\left[I_{\left\{N=n, \tau \leq T_{1}\right\}}\right]=\sum_{n=2}^{\infty} \mathbb{E}\left[I_{\left\{N=n, \tau \leq T_{1}\right\}}\right] \\
& \leq \sum_{n=2}^{\infty} \mathbb{E}\left[I_{\{N \geq n\}} \leq \sum_{n=1}^{\infty} P(N \geq n)-P(N \geq 1)\right. \\
& =\mathbb{E}[N]-1=\beta
\end{aligned}
$$

e a condição $q \rightarrow 0$ é obtida quando $\beta \rightarrow 0$.

Consideremos a probabilidade da primeira perda de demanda condicionada ao tempo de espera para a primeira chegada:

$$
P\left(A^{-} \mid \xi_{1}=x\right)=P\left(\tau<T_{1} \mid \xi_{1}=x\right)=q(x)
$$

Vamos supor que quando o intervalo entre duas demandas cresce, a probabilidade condicional de perda de uma demanda no primeiro período de regeneração diminui. A formulação matemática dessa suposição é o decrescimento monótono de $q(x)$ como função de $x$. Consequentemente,

$$
\begin{aligned}
\mathbb{E}\left[\left(1-e^{-z \xi_{1}}\right) I_{\left\{\tau \leq T_{1}\right\}}\right] & =\int_{0}^{\infty}\left(1-e^{-z x}\right) q(x) d F(x) \\
& \leq \int_{0}^{\infty}\left(1-e^{-z x}\right) d F(x) \int_{0}^{\infty} q(x) d F(x) \\
& =q[1-f(z)],
\end{aligned}
$$

onde a desigualdade acima é devida à Desigualdade de Chebyshev (vide apêndice C) Portanto, concluímos que

$$
\delta=\sup _{z>0} \frac{\mathbb{E}\left[\left(1-e^{-z \xi_{1}}\right) I_{\left\{\tau \leq T_{1}\right\}}\right]}{\mathbb{E}\left[1-e^{-z \xi_{1}}\right]} \leq q .
$$

Assim, se as condições

$$
\begin{array}{ll}
1: & I_{\left\{N=1, \tau \leq T_{1}\right\}} \equiv 0 \\
2: & P\left(A^{-} / \xi_{1}=x\right)=q(x) \downarrow
\end{array}
$$

estão satisfeitas, as condições (2.4) reduzem-se a

$$
q>0, \quad \mathbb{E}[N] \rightarrow 1
$$




\subsection{O sistema $(\mathrm{r}, \mathrm{m}-\mathrm{r})$}

Denotamos por sistema $(r, m-r)$ a um sistema de filas que consta de $r$ unidades de atendimento (ou dispositivos de reparo) e capacidade da fila de espera de $m-r$ lugares. Para este tipo de sistema, a primeira perda de demanda ocorre no instante em que a demanda chega a procura de atendimento e encontra todas as $r$ unidades de reparo e os $m-r$ lugares da fila de espera ocupados. Para aplicar o Teorema 2.2.1 a este sistema particular, observamos, primeiro, se as condições (2.14) estão satisfeitas, reduzindo desta forma as condições do teorema.

Para $m>1, I_{\left\{N=1, \tau \leq T_{1}\right\}} \equiv 0$, isto é, não ocorrerá a perda de uma demanda na primeira chegada após $t_{0}=0$, pois a capacidade do sistema (número de dispositivos de reparo mais número de lugares de espera) é maior ou igual a 2. Segue-se que a primeira das condições de (2.14) está satisfeita.

Para provar que a segunda condição de (2.14) é valida, considere $x_{1}$ e $x_{2}, x_{1}<x_{2}$, valores arbitrários de $\xi_{1}$. Dado que $\nu(t)$ depende do número de demandas presentes no sistema, $\nu(t)$ pode assumir dois valores no instante $x_{1}$, a saber,

$$
\nu(t)= \begin{cases}0 & \text { se o primeiro serviço ja foi completado } \\ 1 & \text { caso contrário. }\end{cases}
$$

No instante $x_{2}$, os valores que $\nu(t)$ pode tomar são os mesmos que para $x_{1}$, com a diferença de que para alguma realização do processo pode ocorrer que o tempo para completar o primeiro serviço seja maior que $x_{1}$ e menor que $x_{2}$, o que implicará que $\nu\left(t_{n}, x_{1}\right) \geq \nu\left(t_{n}, x_{2}\right)$, onde $\left(t_{n}, x\right)=x+\xi_{2}+\cdots+\xi_{n}$.

Assim, $q(x)=P\left(\tau \leq T_{1} \mid \xi_{1}=x\right)$ decresce monótonamente e, portanto, a segunda condição de (2.14) é satisfeita. Desta forma, para que o Teorema 2.2.1 possa ser aplicado, basta que

$$
\mathbb{E}[N] \rightarrow 1, \quad \text { e } \quad q>0
$$


Precisamos achar as condições sob as quais (2.15) é verificada

Considere o sistema $(1, \infty)$ : um dispositivo de reparo e infinitos lugares de espera. Neste sistema não ocorrem perdas de demanda. Denotemos por $\bar{\nu}(t)$ a trajetória deste processo e por $\bar{N}$ a variável aleatória que indica o número de demandas necessárias para uma regeneração de $\bar{\nu}(t)$. Como uma fila que consta de um único servidor se ocupa mais rápido que aquela que tem mais do que um, temos que

$$
\begin{gathered}
\bar{N}(t, w) \geq N(t, w) \quad e \\
\mathbb{E}[\bar{N}] \geq \mathbb{E}[N] \geq 1 .
\end{gathered}
$$

Concluímos assim, que se $\mathbb{E}[\bar{N}] \rightarrow 1$, então $\mathbb{E}[N] \rightarrow 1$ e que as condições para provar o Teorema 2.2.1 passam a ser:

$$
\mathbb{E}[\bar{N}] \rightarrow 1 \quad \text { e } \quad q>0
$$

Para tanto, introduzimos a notação necessária e provamos algumas proposições.

Sejam $F_{n}(x)$ e $G_{n}(x)$ as n-ésimas convoluções das distribuições $F(x)$ e $G(x)$, respectivamente.

Sejam

$$
\begin{gathered}
H(x)=\sum_{n=1}^{\infty} F_{n}(x), \quad \bar{F}_{n}(x)=1-F_{n}(x), \quad \bar{G}_{n}(x)=1-G_{n}(x) \\
t_{n}=\xi_{1}+\xi_{2}+\cdots+\xi_{n}, \quad t_{0}=0, e \\
S_{n}=\eta_{1}+\eta_{2}+\cdots+\eta_{n}, \quad S_{0}=0 .
\end{gathered}
$$

Definimos:

$$
\alpha_{k l}=P\left(S_{k}>t_{l}\right)=\int_{0}^{\infty} P\left(S_{k}>t_{l} / t_{l}=t\right) d F_{l}(t)=\int_{0}^{\infty} \bar{G}_{k}(t) d F_{l}(t),
$$

indicando a probabilidade que o tempo para completar o k-ésimo reparo é maior que o tempo para a l-ésima demanda, $k=1,2, \ldots, l=1,2, \ldots$ 
Sejam

$$
\begin{aligned}
\alpha_{1 l}=\alpha_{l} & =P\left(S_{1}>t_{l}\right)=\int_{0}^{\infty} \bar{G}(t) d F_{l}(t) \quad e \\
\alpha & =\sum_{l=1}^{\infty} \alpha_{l}=\int_{0}^{\infty} \bar{G}(x) d H(x)
\end{aligned}
$$

\section{Lema 2.3.1}

Se

$$
\sum_{l=k-1}^{\infty} \alpha_{k l}=\sum_{l=k-1}^{\infty} P\left(S_{k}>t_{l}\right)<1
$$

para algum $k>1$, então:

$$
\mathbb{E}[. V] \leq \mathbb{E}[\bar{N}] \leq 1+\frac{k \alpha}{1-\sum_{l=k-1}^{\infty} \alpha_{k l}}
$$

PROVA:

Este lema está provado no Apêndice D.

\section{Lema 2.3.2}

Considere $\sum_{l=k}^{\infty} \alpha_{k l}=\sum_{l=k}^{\infty} P\left(S_{k}>t_{l}\right)$.

Para $s \in N$ tal que $k s \leq l<k(s+1)$, então:

$$
1-\alpha_{k l} \geq 1-\alpha_{k, k s} \geq\left(1-\alpha_{s}\right)^{k} \geq 1-k \alpha_{s}
$$

PROVA:

Para a primeira desigualdade do lado esquerdo, observe que se $k s \leq l$, então $t_{k s} \leq t_{l}$, e

$$
1-\alpha_{k l}=P\left(S_{k} \leq t_{l}\right) \geq P\left(S_{k} \leq t_{k s}\right)=1-\alpha_{k, k s}
$$

Para a desigualdade seguinte. observe que, para todo $k$, a ocorrência do evento $\left\{\eta_{1} \leq\right.$ $\left.\xi_{s}, \eta_{2} \leq \xi_{2 s}, \ldots, \eta_{k} \leq \xi_{k s}\right\}$ implica na ocorrência do evento $\left\{S_{k} \leq t_{k s}\right\}$, considerando que $\sum_{i=1}^{k} \xi_{i s}=t_{k s}, \sum_{i=1}^{k} \eta_{i}=S_{k}$. Como os eventos $\left\{\eta_{i} \leq \xi_{i s}\right\}, i=1 \ldots k$, são independentes e têm mesma probabilidade $P\left(S_{1} \leq t_{s}\right)$, temos:

$$
1-\alpha_{k, k s}=P\left(S_{k} \leq t_{k s}\right) \geq\left(P\left(S_{1} \leq t_{s}\right)\right)^{k}=\left(1-\alpha_{s}\right)^{k}
$$


Observemos ainda que para $a \in[0,1],(1-a)^{k} \geq 1-k a$. Então, $\left(1-\alpha_{s}\right)^{k} \geq 1-k \alpha_{s}$ e a desigualdade do lado direito está provada.

Do lema 2.2 .4 observamos que $\alpha_{k l} \leq k \alpha_{s}$ e que

$$
\sum_{l=k}^{\infty} \alpha_{k l}=\alpha_{k k}+\alpha_{k, k+1}+\ldots \leq k \alpha_{s}+k \alpha_{s+1}+\ldots=k \sum_{l=s}^{\infty} \alpha_{l} .
$$

Como $k \geq 1$, temos

$$
\sum_{l=k}^{\infty} \alpha_{k l} \leq k \sum_{l=s}^{\infty} \alpha_{l} \leq k \sum_{l=1}^{\infty} \alpha_{l} \leq k^{2} \alpha
$$

\section{Lema 2.3.3}

Para um inteiro positivo arbitrário $k$,

$$
\alpha_{k+1, k} \leq 1-\left[\int_{0}^{\infty} G\left[\frac{k-1}{k} x\right] d F(x)\right]^{2 k}
$$

\section{PROVA}

É fácil observar que a ocorrência do evento $\left\{S_{k} \leq \frac{k-1}{k} t_{k}, \eta_{k+1} \leq \frac{t_{k}}{k}\right\}$ implica na ocorrência do evento $\left\{S_{k+1} \leq t_{k}\right\}$ e, portanto,

$$
P\left(S_{k+1} \leq t_{k}\right) \geq P\left(S_{k} \leq \frac{k-1}{k} t_{k}, \eta_{k+1} \leq \frac{t_{k}}{k}\right)
$$

Como $G_{k}$ e $G$ são funções monótonas crescentes e não negativas, da Desigualdade de Chebyshev concluímos que

$$
\begin{aligned}
P\left(S_{k+1} \leq t_{k}\right)=1-\alpha_{k+1, k} & =\int_{0}^{\infty} G_{k+1}(x) d F_{k}(x) \\
& \geq \int_{0}^{\infty} G_{k}\left(\frac{k-1}{k} x\right) G\left(\frac{x}{k}\right) d F_{k}(x) \\
& \geq \int_{0}^{\infty} G_{k}\left(\frac{k-1}{k} x\right) d F_{k}(x) \int_{0}^{\infty} G\left(\frac{x}{k}\right) d F_{k}(x)
\end{aligned}
$$

Considerando uma integração por partes e mudança de variáveis, temos:

$$
1-\alpha_{k+1, k} \geq \int_{0}^{\infty} G_{k}\left(\frac{k-1}{k} x\right) d F_{k}(x) \int_{0}^{\infty} \bar{F}_{k}(k x) d G(x) .
$$


Por outro lado, o evento $\left\{\eta_{1} \leq \frac{k-1}{k} \xi_{1}, \eta_{2} \leq \frac{k-1}{k} \xi_{2}, \ldots, \eta_{k} \leq \frac{k-1}{k} \xi_{k}\right\}$ implica no evento $\left\{S_{k} \leq \frac{k-1}{k} t_{k}\right\}$. Pela independência dos $\eta_{i}$ e dos $\xi_{i}$ temos:

$$
\int_{0}^{\infty} G_{k}\left(\frac{k-1}{k} x\right) d F_{k}(x) \geq\left[\int_{0}^{\infty} G\left(\frac{k-1}{k} x\right) d F(x)\right]^{k} .
$$

Da mesma maneira, o evento $\left\{\xi_{1} \geq \eta_{1}, \xi_{2} \geq \eta_{1}, \ldots, \xi_{k} \geq \eta_{1}\right\}$ implica no evento $\left\{t_{k} \geq k \eta_{1}\right\}$ e temos:

$$
\int_{0}^{\infty} \bar{F}_{k}(k x) d G(x) \geq\left[\int_{0}^{\infty} \bar{F}(x) d G(x)\right]^{k}
$$

Das duas desigualdades acima e por (2.20) concluimos:

$$
1-\alpha_{k+1, k} \geq\left[\int_{0}^{\infty} G\left(\frac{k-1}{k} x\right) d F(x)\right]^{k}\left[\int_{0}^{\infty} \bar{F}(x) d G(x)\right]^{k}
$$

Se consideramos a integração por partes, podemos expressar

$$
\int_{0}^{\infty} \bar{F}(x) d G(x)=\int_{0}^{\infty} G(x) d F(x)
$$

e como $G$ e $F$ são funções monótonas crescentes e não negativas, garantimos que

$$
\int_{0}^{\infty} \bar{F}(x) d G(x) \geq \int_{0}^{\infty} G\left(\frac{k-1}{k} x\right) d F(x),
$$

pois

$$
\frac{k-1}{k} x \leq x \text { implica } G\left(\frac{k-1}{k} x\right) \leq G(x) .
$$

Substituindo em (2.21), obtemos que

$$
1-\alpha_{k+1, k} \geq\left[\int_{0}^{\infty} G\left(\frac{k-1}{k} x\right) d F(x)\right]^{2 k}
$$

o que prova o lema.

Usaremos os Lemas 2.3.1 e 2.3.3 para provar o Teorema 2.3.1. 


\section{Teorema 2.3.1}

Se as distribuições $F$ e $G$ e os números $r$ e $m$ no sistema $(r, m-r), m>1$, variam de tal forma que

1. $\alpha \rightarrow 0$

2. $\alpha_{m}>0 e$

3. Existem números positivos $\epsilon_{0}$ e $p_{0}$ tais que

$$
P\left(\eta \leq\left(1-\epsilon_{0}\right) \xi\right) \geq p_{0}
$$

então $q>0$ e $\mathbb{E}[N] \rightarrow 1$, isto é, as condições (2.4) estão satisfeitas e o Teorema 2.2.1 vale para este sistema.

\section{PROVA}

Observe que o evento $\left\{S_{1}>t_{m}\right\}$ implica no evento $\left\{\tau \leq T_{1}\right\}$, correspondente a perda de uma demanda no primeiro período de regeneração, de maneira que

$$
P\left(\tau \leq T_{1}\right)=q \geq P\left(S_{1}>t_{m}\right)=\alpha_{m}
$$

Tomemos um $k>\frac{1}{\epsilon_{0}}+1$. Desde que $\epsilon_{0}>\frac{1}{k-1}$, temos:

$$
1-\epsilon_{0}<1-\frac{1}{k-1}=\frac{k-2}{k-1} \text {. }
$$

Como G é uma função monótona crescente,

$$
\begin{gathered}
p_{0} \leq P\left(\eta \leq\left(1-\epsilon_{0}\right) \xi\right) \leq P\left(\eta \leq \frac{k-2}{k-1} \xi\right)=\int_{0}^{\infty} G\left(\frac{k-2}{k-1} x\right) d F(x), \quad \text { donde } \\
1-p_{0}^{2 k-2} \geq 1-\left[\int_{0}^{\infty} G\left(\frac{(k-1)-1}{k-1} x\right) d F(x)\right]^{2(k-1)} .
\end{gathered}
$$

Pelo Lema 2.3.3,

$$
1-p_{0}^{2(k-1)} \geq 1-\left[\int_{0}^{\infty} G\left(\frac{(k-1)-1}{k-1} x\right) d F(x)\right]^{2(k-1)} \geq \alpha_{k, k-1}
$$


Na observação 2.1 do Teorema 2.2.1, obtivemos que $0<q<\mathbb{E}[N-1] \leq \mathbb{E}[\bar{N}-1]$, onde $\bar{N}$ representa o número de chegadas no período de regeneração de $\bar{\nu}(t)$, quando consideramos o sistema $(1, \infty)$. Agora, considerando (2.18) e (2.23), obtemos:

$$
1-\alpha_{k, k-1}-\sum_{l=k}^{\infty} \alpha_{k l} \geq p_{0}^{2 k-2}-k^{2} \alpha
$$

Logo,

$$
\frac{k \alpha}{1-\alpha_{k, k-1}-\sum_{l=k}^{\infty} \alpha_{k l}} \leq \frac{k \alpha}{p_{0}^{2 k-2}-k^{2} \alpha}
$$

e, portanto,

$$
1 \leq \mathbb{E}[N] \leq \mathbb{E}[\bar{N}] \leq 1+\frac{k \alpha}{1-\sum_{l=k-1}^{\infty} \alpha_{k l}}=1+\frac{k \alpha}{1-\alpha_{k, k-1}-\sum_{l=k}^{\infty} \alpha_{k l}} \leq 1+\frac{k \alpha}{p_{0}^{2 k-2}-k^{2} \alpha}
$$

Como, por hipótese, $\alpha \rightarrow 0$, temos:

$$
1 \leq \mathbb{E}[N] \leq \mathbb{E}[\bar{N}] \leq 1
$$

o que prova o teorema.

O Teorema 2.3.1 é válido sob condições mais fracas, quando consideramos $m$ dispositivos de reparo sem fila de espera, isto é, o sistema $(m, 0)$. Neste caso, obtemos uma estimativa interessante para $\mathbb{E}[N]$.

\section{Lema 2.3.4}

Se

$$
\alpha=\sum_{k=1}^{\infty} \alpha_{k}=\sum_{k=1}^{\infty} P\left(S_{1}>t_{k}\right)=\int_{0}^{\infty} \bar{G}(x) d H(x)<\infty,
$$

onde $H(x)=\sum_{n=1}^{\infty} F_{n}(x)$,

então, para o sistema $(m, 0)$,

$$
\mathbb{E}[N] \leq \frac{1}{\prod_{k=1}^{\infty}\left(1-\alpha_{k}\right)}
$$




\section{PROVA}

Considere o sistema com infinitos servidores $(\infty, 0)$, no qual não ocorrem perdas de demanda. Denotemos por $\widetilde{N}$ o número de chegadas num período ocupado e por $\widetilde{\nu}(t)$ ao número de servidores ocupados no tempo $t$.

Os processos de renovação a tempo discreto são análogos às cadeias de Markov com os estados 0 e 1 , definidos pela ocorrência ou não do evento $E=\left\{\right.$ renovação no instante $\left.t_{n}^{-}\right\}$. Definimos uma sequência de variáveis aleatórias $\left(\chi_{n}\right)_{n \geq 1}$, onde $\chi_{n}$ denota a ocorrência ou não de uma renovação em $t_{n}^{-}$, isto é,

$$
\chi_{n}= \begin{cases}1 & \text { se ocorre uma renovação em } t_{n}^{-} \\ 0 & \text { caso contrário }\end{cases}
$$

Seja

$\pi_{n}$ : probabilidade da primeira renovação ocorrer no instante da $n$-ésima demanda, isto é,

$$
\pi_{n}=P\left(\chi_{n}=1, \chi_{m}=0, m=1, \ldots, n-1 / \chi_{0}=1\right)
$$

Claramente,

$$
\pi_{n}=P(\widetilde{N}=n)
$$

Seja

$p_{n}$ : probabilidade que o evento E ocorra no instante da n-ésima demanda sem levar em consideração se houve alguma renovação em alguma demanda anterior, isto é,

$$
p_{n}=P\left(\tilde{\nu}\left(t_{n}^{-}\right)=0\right)=P\left(\chi_{n}=1 \mid \chi_{0}=1\right), n=1,2, \ldots,
$$

$\operatorname{com} p_{0}=1$.

Como em uma Cadeia de Markov, os $\pi_{n}$ e $p_{n}$ se relacionam de maneira que

$$
\begin{gathered}
p_{n}=\sum_{l=1}^{n} \pi_{l} p_{n-l} e \\
\pi_{n}=p_{n}-\sum_{l=1}^{n-1} \pi_{l} p_{n-l},
\end{gathered}
$$


isto é, existe uma relação entre os $\pi_{i}^{\prime} s$ e os $p_{i}^{\prime} s$.

Se para $|z| \leq 1, p(z)$ e $\pi(z)$, são as funções geradoras de probabilidade de $\left(p_{n}\right)_{n \geq 0} \mathrm{e}$ $\left(\pi_{n}\right)_{n \geq 1}$ respectivamente, isto é,

$$
\begin{aligned}
& p(z)=\sum_{n=0}^{\infty} p_{n} z^{n} e \\
& \pi(z)=\sum_{n=1}^{\infty} \pi_{n} z^{n},
\end{aligned}
$$

podemos provar que

$$
p(z)=\frac{1}{1-\pi(z)}
$$

ou, alternativamente,

$$
\pi(z)=1-\frac{1}{p(z)}
$$

Assim,

$$
\frac{1-\pi(z)}{1-z}=\frac{1}{(1-z) p(z)}
$$

Observe que

$$
\left(1-\alpha_{i}\right)=P\left(S_{1} \leq t_{i}\right)=\int_{0}^{\infty} G(t) d F_{i}(t) .
$$

Logo,

$$
\begin{aligned}
\prod_{i=1}^{n}\left(1-\alpha_{i}\right) & =\prod_{i=1}^{n} \int_{0}^{\infty} G(t) d F_{i}(t)=\prod_{i=1}^{n} \int_{0}^{\infty} \bar{F}_{i}(t) d G(t) \\
& \leq \int_{0}^{\infty} \prod_{i=1}^{n} \bar{F}_{i}(t) d G(t) \\
& =\int_{0}^{\infty} \prod_{i=1}^{n} P\left(\xi_{i}>t\right) d G(t) \\
& =\int_{0}^{\infty} P\left(\xi_{1}>t, \xi_{2}>t, \ldots, \xi_{n}>t\right) d G(t) \\
& =\int_{0}^{\infty} P\left(\xi_{1}>t, \xi_{2}>t, \ldots, \xi_{n}>t \mid S_{1}=t\right) d G(t) \\
& =P\left(\xi_{1}>S_{1}, \xi_{2}>S_{1}, \ldots, \xi_{n}>S_{1}\right) \\
& \leq P\left(\eta_{1} \leq \xi_{1}+\xi_{2}+\cdots+\xi_{n}, \eta_{2} \leq \xi_{2}+\cdots+\xi_{n}, \ldots, \eta_{n} \leq \xi_{n}\right)=p_{n}
\end{aligned}
$$


Assim,

$$
p_{n} \geq\left(1-\alpha_{1}\right)\left(1-\alpha_{2}\right) \ldots\left(1-\alpha_{n}\right) \geq \prod_{n=1}^{\infty}\left(1-\alpha_{n}\right)>0 .
$$

Podemos calcular $p_{n}$, considerando que os $\eta_{i}$ são independentes:

$$
\begin{aligned}
p_{n} & =P\left(\eta_{1} \leq \xi_{1}+\cdots+\xi_{n}, \eta_{2} \leq \xi_{2}+\cdots+\xi_{n}, \ldots, \eta_{n} \leq \xi_{n}\right) \\
& =\int_{0}^{\infty} P\left(\eta_{1} \leq \xi_{1}+\cdots+\xi_{n}, \ldots, \eta_{n} \leq \xi_{n} / \xi_{n}=x_{n}\right) d F\left(x_{n}\right) \\
& =\int_{0}^{\infty} P\left(\eta_{1} \leq \xi_{1}+\cdots+x_{n}, \ldots, \eta_{n} \leq x_{n}\right) d F\left(x_{n}\right) .
\end{aligned}
$$

Aplicando o procedimento acima n vezes e levando em consideração a independência entre os $\eta_{i}$ e os $\xi_{i}$, concluímos que:

$$
\begin{aligned}
p_{n} & =\int_{0}^{\infty} \ldots \int_{0}^{\infty} P\left(\eta_{1} \leq x_{1}+\cdots+x_{n}, \eta_{2} \leq x_{2}+\cdots+x_{n}, \ldots, \eta_{n} \leq x_{n}\right) d F\left(x_{1}\right) \ldots d F\left(x_{n}\right) \\
& =\int_{0}^{\infty} \ldots \int_{0}^{\infty} G\left(x_{1}+\cdots+x_{n}\right) G\left(x_{2}+\cdots+x_{n}\right) \ldots G\left(x_{n}\right) d F\left(x_{1}\right) \ldots d F\left(x_{n}\right) .
\end{aligned}
$$

Observamos que $p_{n}$ decresce monotonicamente e, por (2.31), provamos a existência do limite de $\left\{p_{n}\right\}_{n \geq 1}$. Temos, portanto, que

$$
\lim _{n \rightarrow \infty} p_{n}=p \geq \prod_{n=1}^{\infty}\left(1-\alpha_{n}\right) .
$$

Como definido anteriormente,

$$
\pi_{n}=P(\widetilde{N}=n) \quad e \quad \pi(z)=\sum_{n=1}^{\infty} \pi_{n} z^{n}
$$

Tomando limite em (2.30) obtemos que

$$
\mathbb{E}[\widetilde{N}]=\pi^{\prime}(1)=\lim _{z \rightarrow 1} \frac{1-\pi(z)}{1-z}=\frac{1}{\lim _{z \rightarrow 1^{-}}(1-z) p(z)}
$$

e, consequentemente,

$$
1 \leq \mathbb{E}[N] \leq \mathbb{E}[\widetilde{N}]=\frac{1}{\lim _{z \rightarrow 1^{-}}(1-z) p(z)}
$$


Provaremos que $\lim _{n \rightarrow \infty} p_{n}=p=\lim _{z \rightarrow 1^{-}}(1-z) p(z)$.

Se $\lim _{n \rightarrow \infty} p_{n}=p$ então, para todo $\epsilon>0$, existe $M$ tal que se $n>M,\left|p-p_{n}\right|<\epsilon / 2$. Como $|z|<1$, temos que

$$
(1-z) \sum_{n=0}^{\infty} z^{n}=(1-z) \frac{1}{1-z}=1
$$

Assim,

$$
\begin{aligned}
\left|(1-z) \sum_{n=0}^{\infty} p_{n} z^{n}-p\right| & =\left|(1-z) \sum_{n=0}^{\infty} p_{n} z^{n}-p(1-z) \sum_{n=0}^{\infty} z^{n}\right| \\
& =\left|(1-z) \sum_{n=0}^{\infty}\left(p_{n}-p\right) z^{n}\right| \\
& \leq(1-z) \sum_{n=0}^{M}\left|p_{n}-p\right| z^{n}+(1-z) \sum_{n=M+1}^{\infty}\left|p_{n}-p\right| z^{n}
\end{aligned}
$$

Como $\sum_{n=0}^{M}\left|p_{n}-p\right| z^{n}$ é limitada, aplicando o limite em $z$, obtemos que

$$
(1-z) \sum_{n=0}^{M}\left|p_{n}-p\right| z^{n} \rightarrow 0 .
$$

Portanto,

$$
\left|(1-z) \sum_{n=0}^{\infty} p_{n} z^{n}-p\right| \leq \epsilon / 2+\epsilon / 2=\epsilon,
$$

provando que

$$
\lim _{z \rightarrow 1^{-}}(1-z) p(z)=p
$$

Isto, junto com (2.31) e (2.32). mostra que

$$
1 \leq \mathbb{E}[N] \leq \mathbb{E}[\widetilde{N}] \leq \pi^{\prime}(1)=\frac{1}{p} \leq \frac{1}{\prod_{n=1}^{\infty}\left(1-\alpha_{n}\right)}
$$

e, portanto, o lema está provado.

\section{Lema 2.3.5}

Se $\alpha<1$, para o sistema $(m, 0)$, então, do Lema anterior segue que

$$
\mathbb{E}[\widetilde{N}] \leq \frac{1}{1-\alpha}
$$




\section{PROVA}

Obtivemos acima que $\mathbb{E}[\widetilde{N}] \leq \frac{1}{\prod_{n=1}^{\infty}\left(1-\alpha_{n}\right)}$, precisamos então provar que

$$
\prod_{n=1}^{\infty}\left(1-\alpha_{n}\right) \geq 1-\sum_{n=1}^{\infty} \alpha_{n}
$$

A prova pode ser feita por indução:

para $n=1$, a desigualdade é verdadeira, suponha que é válida também para $n=k$ :

$$
\prod_{i=1}^{k}\left(1-\alpha_{i}\right) \geq 1-\sum_{i=1}^{k} \alpha_{i}
$$

Considere agora, $n=k+1$

$$
\begin{aligned}
\prod_{i=1}^{k+1}\left(1-\alpha_{i}\right) & =\prod_{i=1}^{k}\left(1-\alpha_{i}\right)\left(1-\alpha_{k+1}\right) \\
& \geq\left(1-\sum_{i=1}^{k} \alpha_{i}\right)\left(1-\alpha_{k+1}\right) \\
& \geq 1-\sum_{i=1}^{k} \alpha_{i}-\alpha_{k+1}+\alpha_{k+1} \sum_{i=1}^{k} \alpha_{i} \\
& \geq 1-\sum_{i=1}^{k+1} \alpha_{i}
\end{aligned}
$$

Aplicando o limite na desigualdade acima, obtemos a expressão desejada. Isto nos leva ao seguinte teorema:

\section{Teorema 2.3.2}

Se $\alpha_{m}>0$, para o sistema $(m, 0)$, e se $\alpha \rightarrow 0$, então $\mathbb{E}[\widetilde{N}] \rightarrow 1$.

\section{PROVA}

A prova deste teorema segue como consequencia imediata dos lemas anteriores. Isto significa que para o sistema $(m, 0)$, o Teorema 2.2.1 é válido. 


\section{Conclusões}

Nesta dissertação enfatizamos o estudo de sistemas reparáveis considerando que as distribuições envolvidas são arbitrárias, o que é de singular importância na Teoría de Confiabilidade desde que o comportamento de sistemas na prática, obedecem a este tipo de situação.

Em um segundo momento, estudamos a confiabilidade assintótica de sistemas com "reparos rápidos" atraves de Teoría das Filas, o que não é uma abordagem usual.

Convem ressaltar que a condição de "reparo rápido" é amplamente usada no decorrer do trabalho e está imersa nas condições de convergência. Por exemplo, a hipótese de $q \rightarrow 0$ no Teorema 2.2.1 ( $q$ representa a probabilidade do evento "perda de uma demanda antes de uma renovação"), indica que a probabilidade de uma falha ocorrer antes do fim de um reparo é muito pequena, a hipótese de $N \rightarrow 1$ no mesmo teorema ( $N$ é a variável aleatória que indica o número de demandas necessárias para a primeira regeneração), indica que a segunda demanda encontrará o sistema desocupado com probabilidade um. $\delta \rightarrow 0$ no mesmo teorema $\left(\delta=\sup _{x>0} \frac{E\left[\left(1-e^{-z \xi_{1}}\right) I_{\left.\tau \leq T_{1}\right]}\right]}{E\left[1-e^{-z \xi_{1}}\right]}\right)$, implica que a função indicadora do evento "perda de uma demanda antes da primeira renovação" é zero com probabilidade um. $\alpha \rightarrow 0$ no Teorema 2.3.1, indica que a probabilidade de um reparo demorar mais do que as proximas chegadas é próxima de zero.

Concluímos que sob hipóteses adequadas, a convergência da distribuição do tempo de vida de um sistema, é para uma distribuição exponencial. 


\section{Apêndice A}

\section{Lemas para o Processo regenerativo}

\section{de duas fases}

Lema A.0.6

$$
\mathbb{E}\left[\tilde{\eta}_{t}\right]=\frac{a_{1} p \lambda t+a_{2}}{p}
$$

onde $a_{1}=(1-p) \mathbb{E}\left[\eta_{n}^{(+)}\right]$e $a_{2}=p \mathbb{E}\left[\eta_{n}^{(-)}\right]$

PROVA

Usando a notação acima, temos que:

$$
\begin{aligned}
\mu^{*} & =\mathbb{E}\left(\eta_{n}\right)=\mathbb{E}\left[\eta_{n}^{(+)}\left(1-\chi_{n}\right)+\eta_{n}^{(-)} \chi_{n}\right] \\
& =(1-p) \mathbb{E}\left[\eta_{n}^{(+)}\right]+p \mathbb{E}\left[\eta_{n}^{(-)}\right]=a_{1}+a_{2} \\
& \leq \mathbb{E}\left[\eta_{n}^{0}\right]=\mu_{0} .
\end{aligned}
$$

Definamos $\bar{F}_{t}(x)$ como sendo a probabilidade condicional de que $\tilde{\eta}$ exceda $x$ dado que $\xi=t$ (em geral, o período ocupado não é independente do período livre anterior):

$$
\bar{F}_{t}(x)=P(\tilde{\eta} \geq x \mid \xi=t)=P\left(\tilde{\eta}_{t} \geq x\right)
$$

Como os períodos livres seguem uma distribuição exponencial, o número de períodos livres até a ocorrência do evento $A$, que indica a falha do sistema, tem distribuição de Poisson 
de parâmetro $\lambda q$,onde $q=1-p$, e assim,

$$
\bar{F}_{t}(x)=\sum_{n=1}^{\infty} \bar{\Phi}_{n}(x) \frac{(\lambda q t)^{n-1}}{(n-1) !} e^{-\lambda q t}
$$

onde

$$
\bar{\Phi}_{n}(x)=P\left(\eta_{1}^{(+)}+\cdots+\eta_{n-1}^{(+)}+\eta_{n}^{(-)} \geq x\right)=P(\tilde{\eta} \geq x) .
$$

Analisamos o comportamento de $\bar{F}_{t}(x)$ derivando a expressão acima:

$$
\begin{aligned}
\frac{d}{d t} \bar{F}_{t}(x) & =\sum_{n=1}^{\infty} \frac{\bar{\Phi}_{n}(x)}{(n-1) !}\left[(\lambda q)^{n-1}(n-1) t^{n-2} e^{-\lambda q t}-(\lambda q t)^{n-1} \lambda q e^{-\lambda q t}\right] \\
& =\lambda q\left[\sum_{m=1}^{\infty} e^{-\lambda q t} \frac{(\lambda q t)^{m-1}}{(m-1) !} \bar{\Phi}_{m+1}(x)-\sum_{n=1}^{\infty} e^{-\lambda q t} \frac{(\lambda q t)^{n-1}}{(n-1) !} \bar{\Phi}_{n}(x)\right] \\
& =\lambda q \sum_{n=1}^{\infty}\left[\bar{\Phi}_{n+1}(x)-\bar{\Phi}_{n}(x)\right] e^{-\lambda q t} \frac{(\lambda q t)^{n-1}}{(n-1) !}
\end{aligned}
$$

Desde que $\bar{\Phi}_{n+1}(x) \geq \bar{\Phi}_{n}(x)$, concluimos que $\frac{d}{d t} \bar{F}_{t}(x) \geq 0$ e que $\bar{F}_{t}(x)$ é monótona crescente em $t$.

Integrando em (A.2), obtemos:

$$
\begin{aligned}
\mathbb{E}\left[\tilde{\eta}_{t}\right] & =\int_{0}^{\infty} \bar{F}_{t}(x) d x=\int_{0}^{\infty} \sum_{n=1}^{\infty} \bar{\Phi}_{n}(x) \frac{(\lambda q t)^{n-1}}{(n-1) !} e^{-\lambda q t} \\
& =\sum_{n=1}^{\infty} \mathbb{E}[\tilde{\eta}] \frac{(\lambda q t)^{n-1}}{(n-1) !} e^{-\lambda q t} \\
& =\sum_{n=1}^{\infty}\left[(n-1) \frac{a_{1}}{q}+\frac{a_{2}}{p}\right] \frac{(\lambda q t)^{n-1}}{(n-1) !} e^{-\lambda q t} \\
& =\frac{a_{1}}{q}(\lambda q t)+\frac{a_{2}}{p}
\end{aligned}
$$

Desta maneira, podemos expressar $\mathbb{E}\left[\tilde{\eta}_{t}\right]$ como

$$
\mathbb{E}\left[\tilde{\eta}_{t}\right]=\frac{a_{1} p \lambda t+a_{2}}{p}
$$

Lema A.0.7

$$
\int_{0}^{t} \lambda p e^{-\lambda p x} \bar{F}_{x}(t-x) d x \leq \lambda \mu^{*}
$$




\section{PROVA}

Se observamos $\int_{0}^{t} \lambda p e^{-\lambda p x} \bar{F}_{x}(t-x) d x$ como:

$J=\int_{0}^{t} \lambda p e^{-\lambda p x} \bar{F}_{x}(t-x) d x=\int_{0}^{\alpha} \lambda p e^{-\lambda p x} \bar{F}_{x}(t-x) d x+\int_{\alpha}^{t} \lambda p e^{-\lambda p x} \bar{F}_{x}(t-x) d x, \quad 0 \leq \alpha \leq t$, temos $J=J_{1}+J_{2}$, onde

$$
\begin{aligned}
& J_{1}=\int_{0}^{\alpha} \lambda p e^{-\lambda p x} \bar{F}_{x}(t-x) d x, \\
& J_{2}=\int_{\alpha}^{t} \lambda p e^{-\lambda p x} \bar{F}_{x}(t-x) d x .
\end{aligned}
$$

Pela Desigualdade de Markov temos que:

$$
\bar{F}_{x}(t-x)=P\left(\tilde{\eta}_{x} \geq t-x\right) \leq \frac{\mathbb{E}\left[\tilde{\eta}_{x}\right]}{t-x} .
$$

Logo, por (A.4),

$$
J_{1} \leq \int_{0}^{\alpha} \lambda p e^{-\lambda p x} \frac{\mathbb{E}\left[\tilde{\eta}_{x}\right]}{t-x} d x=\int_{0}^{\alpha} \lambda p e^{-\lambda p x}\left[\frac{a_{1} p \lambda x+a_{2}}{p(t-x)}\right] d x
$$

Considere agora as seguintes transformações: $z=\lambda p x, w=\lambda p t$, e $d=\lambda p \alpha$. Então, temos o seguinte limite para a expressão de $J_{1}$ :

$$
J_{1} \leq \lambda \int_{0}^{d} e^{-z}\left[\frac{a_{1} z+a_{2}}{(w-z)}\right] d z
$$

Desde que $\bar{F}_{x}(t-x)$ é monótona crescente, concluimos que

$$
\begin{aligned}
J_{2} & \leq \lambda p e^{-\lambda p \alpha} \int_{\alpha}^{t} \bar{F}_{x}(t-x) d x \\
& \leq \lambda p e^{-\lambda p \alpha} \int_{\alpha}^{t} \bar{F}_{t}(x) d x \leq \lambda p e^{-\lambda p \alpha} \int_{0}^{\infty} \bar{F}_{t}(x) d x \\
& =\lambda p e^{-\lambda p \alpha}\left[\frac{a_{1} \lambda p t+a_{2}}{p}\right]
\end{aligned}
$$

e, considerando as transformações de variáveis feitas acima, temos que

$$
J_{2} \leq \lambda e^{-d}\left(a_{1} w+a_{2}\right)
$$


Desta maneira, por (A.6) e (A.7), segue que

$$
J=J_{1}+J_{2} \leq \lambda\left[\int_{0}^{d} e^{-z}\left[\frac{a_{1} z+a_{2}}{(w-z)}\right] d z+e^{-d}\left(a_{1} w+a_{2}\right)\right]
$$

com $0 \leq d \leq w$.

Se $w=1$, substituímos $d=0$ em (A.8) e

$$
J \leq \lambda\left(a_{1}+a_{2}\right)=\lambda \mu^{*}
$$

Se $w \geq 1$, substituímos $d=w-1$ em (A.8),

$$
J(w) \leq \lambda\left[\int_{0}^{w-1} e^{-z}\left[\frac{a_{1} z+a_{2}}{(w-z)}\right] d z+e^{-(w-1)}\left(a_{1} w+a_{2}\right)\right] .
$$

Usando a Regra de Leibnitz, temos

$$
J^{\prime}(w)=-\lambda\left[\int_{0}^{w-1} e^{-z}\left[\frac{a_{1} z+a_{2}}{(w-z)^{2}}\right] d z \leq 0 .\right.
$$

Então J(w) é uma função decrescente e, portanto,

$$
\max (J(w))=J(1)=\lambda\left(a_{1}+a_{2}\right)=\lambda \mu^{*}
$$

o que indica que

$$
J(w) \leq \lambda \mu^{*},
$$

e o lema está demonstrado. 


\section{Apêndice B}

\section{Transformada de Laplace Racional}

Se o comportamento do sistema é descrito por um processo de Markov homogêneo com o número finito de estados, as configurações dos diversos estados do sistema no tempo são caracterizadas por variáveis aleatórias com transformadas de Laplace racionais que se classificam da seguinte maneira:

$$
\begin{gathered}
K_{0}=\left\{x: \mathbb{E}\left[e^{-z x}\right]=\prod_{k=1}^{n}\left(1-\alpha_{k} z\right)^{-1}=\frac{1}{1+z+a_{2} z^{2}+\cdots+a_{n} z^{n}}\right\} \\
K_{1}=\left\{x: \mathbb{E}\left[e^{-z x}\right]=\frac{1}{1+z+a_{2} z^{2}+\cdots+a_{n} z^{n}}\right\} \\
K_{2}=\left\{x: \mathbb{E}\left[e^{-z x}\right]=\frac{1+b_{1} z+b_{2} z^{2}+\cdots+b_{m} z^{m}}{1+z+a_{2} z^{2}+\cdots+a_{n} z^{n}}\right\}
\end{gathered}
$$

Observe que

$$
K_{0} \subset K_{1} \subset K_{2}
$$

Seja $x(t)$ um processo de Markov homogêneo com um número finito de estados $0,1,2, \ldots, N$. Isto significa que para qualquer conjunto de estados $i_{1}, i_{2}, \ldots, i_{k}, i, j, i \neq j$, e para quaisquer momentos $0 \leq t_{1}<t_{2}<\ldots<t_{k}<t<t+h$, a probabilidade condicional

$$
P\left(x(t+h)=j \mid x(t)=i, x\left(t_{1}\right)=i_{1}, \ldots, x\left(t_{k}\right)=i_{k}\right)=P(x(t+h)=j \mid x(t)=i)=\lambda_{i j} h+o(h)
$$


Em confiabilidade, o processo de Markov descreve um sistema cujos tempos de vida e tempos de reparo de cada elemento não dependem dos estado dos outros elementos e tem distribuição exponencial.

Da definição de processo de Markov, observamos que:

1. O intervalo $\xi_{i}$ no qual o processo está no estado $i$, não depende do comportamento do processo fora desse intervalo e tem distribuição exponencial:

$$
P\left(\xi_{i}>t\right)=e^{\lambda_{i i t} t} \text {, onde } \lambda_{i i}=-\sum_{i \neq j} \lambda_{i j}
$$

2. A sequência de estados do processo, forma uma cadeia de Markov com probabilidades de transição:

$$
\pi_{i j}=-\frac{\lambda_{i j}}{\lambda i i}
$$

(se $\lambda_{i i}=0$, o estado $i$ é absorvente e podemos sustituir $\pi_{i j}=0$ ).

Sejam as probabilidades $p_{i}(t)=P(x(t)=i)$ tais que satisfazem a equação diferencial de Kolmogorov:

$$
p_{j}^{\prime}(t)=\sum_{i=0}^{N} \lambda_{i j} p_{i}(t), j=0,1, \ldots, N
$$

Este sistema pode ser representado em uma forma matricial por:

$$
\mathcal{P}^{\prime}(t)=\mathcal{P} \Delta,
$$

onde $\mathcal{P}(t)=\left[P_{0}(t), \ldots, P_{N}(t)\right]$ é o vetor de estados e $\Delta=\left\{\lambda_{i j}\right\}$ é a matriz de transição de probabilidades. Esta matriz tem as seguintes propriedades:

1. $\lambda_{i j} \geq 0$ quando $i \neq j$,

2. $\sum_{j=0}^{N} \lambda_{i j}=0$.

O sistema (B.1), tem solução única se a distribuição inicial do processo é $p_{i}(0)=p_{i 0}$.

A solução de (B.1), pode ser obtida aplicando a transformada de Laplace. Seja

$$
a_{i}(z)=\int_{0}^{\infty} e^{-z t} p_{i}(t) d t
$$


usando as propriedades elementares da transformada de Laplace e de (B.1) obtemos:

$$
-p_{j 0}+z a_{j}(z)=\sum_{i=0}^{N} a_{i}(z) \lambda_{i j}, j=0,1, \ldots, N
$$

A solução do sistema acima, usando a regra de Cramer é dada por

$$
a_{i}(z)=\frac{\Delta_{i}(z)}{\Delta(z)}
$$

onde

$$
\Delta(z)=\left\|z \delta_{i j}-\lambda_{i j}\right\|
$$

$\delta_{i j}$ é o símbolo de Cramer e o numerador em (B.2) é obtida do denominador sustituindo a i-ésima coluna pela coluna da probabilidade inicial $\left\{p_{j 0}\right\}$.

Assim, a representação (B.2) tem a forma da transformada de Laplace Racional. 


\section{Apêndice $\mathrm{C}$}

\section{Desigualdade de Chebyshev}

Suponha que $f(x)$ e $g(x)$ são funções monôtonas não negativas, e que $F(x)$ é uma função de distribuição. Então,

Se ambas as funções crescem ou decrescem monotónicamente,

$$
\int_{-\infty}^{\infty} f(x) g(x) d F(x) \geq \int_{-\infty}^{\infty} f(x) d F(x) \int_{-\infty}^{\infty} g(x) d F(x)
$$

PROVA

Considere dois argumentos $x$ e $y$,

$$
[f(x)-f(y)][g(x)-g(y)] \geq 0
$$

desenvolvendo esta desigualdade temos:

$$
f(x) g(x)+f(y) g(y)-f(y) g(x)-f(x) g(y) \geq 0 .
$$

Integrando em $x$ temos:

$$
\int_{-\infty}^{\infty} f(x) g(x) d F(x)+f(y) g(y)-f(y) \int_{-\infty}^{\infty} g(x) d F(x)-g(y) \int_{-\infty}^{\infty} f(x) d F(x) \geq 0 .
$$

Integrando em $y$ temos:

$$
\begin{aligned}
\int_{-\infty}^{\infty} f(x) g(x) d F(x)+\int_{-\infty}^{\infty} f(y) g(y) d F(y) & -\int_{-\infty}^{\infty} f(y) d F(y) \int_{-\infty}^{\infty} g(x) d F(x)- \\
& -\int_{-\infty}^{\infty} g(y) d F(y) \int_{-\infty}^{\infty} f(x) d F(x) \geq 0 .
\end{aligned}
$$


Como

$$
\begin{gathered}
\int_{-\infty}^{\infty} f(x) g(x) d F(x)=\int_{-\infty}^{\infty} f(y) g(y) d F(y) . \quad e \\
\int_{-\infty}^{\infty} f(x) d F(x)=\int_{-\infty}^{\infty} f(y) d F(y)
\end{gathered}
$$

concluimos

$$
\int_{-\infty}^{\infty} f(x) g(x) d F(x) \geq \int_{-\infty}^{\infty} f(x) d F(x) \int_{-\infty}^{\infty} g(x) d F(x)
$$

Para o caso quando uma das funções cresce monótonamente e a outra decresce monótonamente, a desigualdade se inverte:

$$
\int_{-\infty}^{\infty} f(x) g(x) d F(x) \leq \int_{-\infty}^{\infty} f(x) d F(x) \int_{-\infty}^{\infty} g(x) d F(x)
$$

Para provar isto, observe que dado os argumentos $x$ e $y$, temos que

$$
[f(x)-f(y)][g(x)-g(y)] \leq 0
$$

e aplicamos o procedimento usado anteriormente. 


\section{Apêndice D}

\section{Lemas para o sistema $(r, m-r)$}

\section{Lema D.0.8}

Se

$$
\sum_{l=k-1}^{\infty} \alpha_{k l}=\sum_{l=k-1}^{\infty} P\left(S_{k}>t_{l}\right)<1
$$

para algum $k>1$, então:

$$
\mathbb{E}[N] \leq \mathbb{E}[\bar{N}] \leq 1+\frac{k \alpha}{1-\sum_{l=k-1}^{\infty} \alpha_{k l}}
$$

PROVA:

Consideremos outra vez o sistema $(1, \infty)$ e denotemos por $\bar{N}_{k}$ à variável aleatória que indica o número de passos no primeiro periodo ocupado sob a condição que em $t_{0-}$ o sistema tem $k$ demandas. Obviamente $\bar{N}_{0}=\bar{N}$.

Para uma realização arbitrária $w$,

$$
\bar{N}_{k} \leq \bar{N}_{11}+\bar{N}_{12}+\cdots+\bar{N}_{1 k}
$$

onde os $\bar{N}_{1 i}$ são independentes e tem a mesma distribuição de $\bar{N}_{1}$.

Uma explicação eurística de (D.2) é a seguinte:

Considere o processo $\nu(t)$ quando entra pela primeira vez no estado $k-1$. O serviço da demanda seguinte será iniciado no instante $t_{\bar{N}_{11}}$, instante em que chega a demanda e não 
imediatamente ao final do último serviço. Assim, o intervalo requerido para o processo ir do estado $k-1$ a $k-2,\left(t_{\bar{N}_{11}}, t_{\bar{N}_{11}+\bar{N}_{12}}\right)$, independe do intervalo $\left(0, t_{\bar{N}_{11}}\right)$. Quando chega uma nova demanda, transferimos o inicio do serviço para o instante $t_{\bar{N}_{11}+\bar{N}_{12}}$ e procedemos da mesma maneira. Estas transferências aumentam o número de passos no periodo ocupado.

Como ilustração gráfica, considere a seguinte realização, supondo que $\nu\left(t_{0}^{-}\right)=2$. $\mathrm{O}$ gráfico superior considera as transferências de inicio dos serviços quando o sistema entra no estado imediato inferior; o gráfico inferior não considera estes tempos vagos dos servidores. Percebemos então, que o número de passos para uma primeira regeneração (nesta realização particular), é menor quando não se consideram as "transferências".

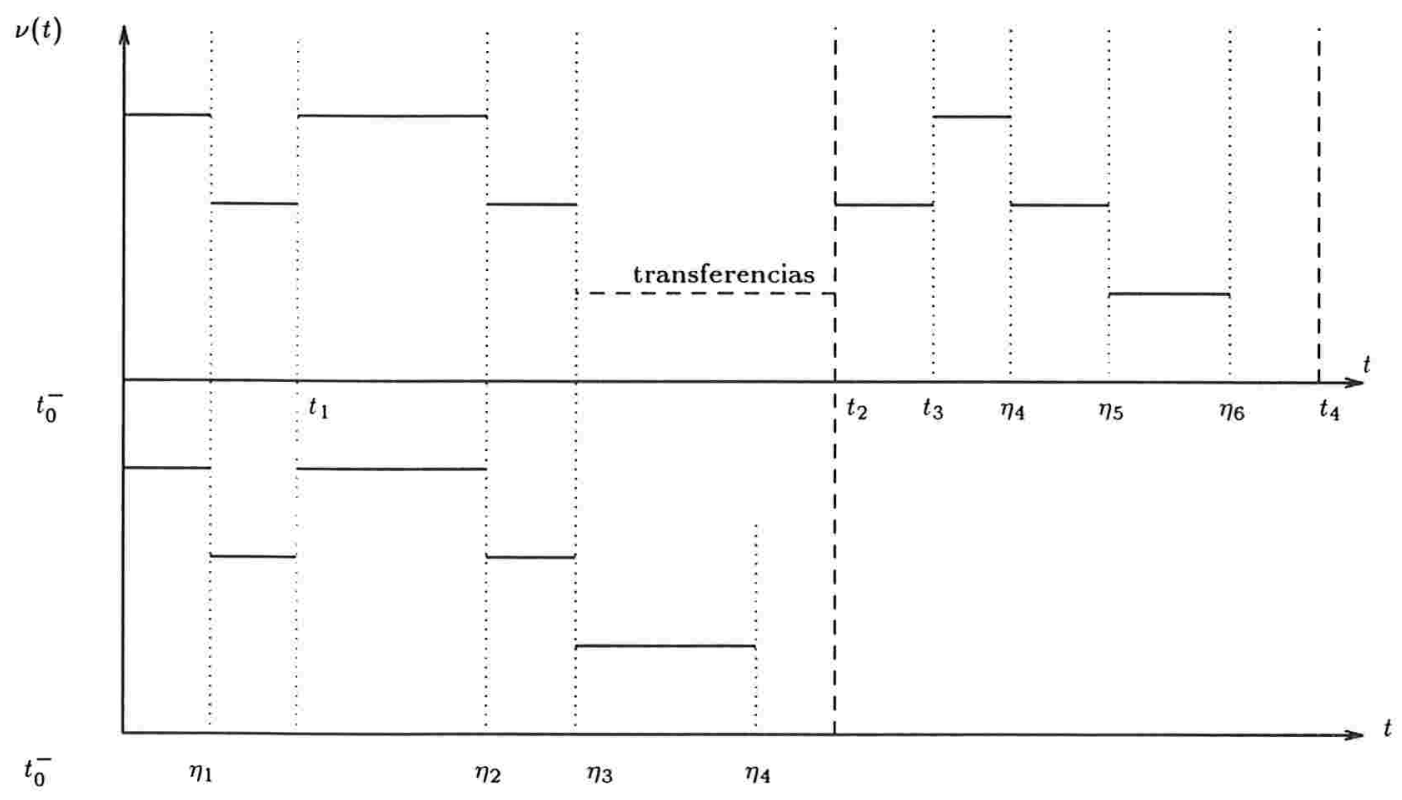

Figura D.1 Comparação entre processos com transferências e sem transferências

De (D.2) temos:

$$
\mathbb{E}\left[\bar{N}_{k}\right] \leq k \mathbb{E}\left[\bar{N}_{1}\right]
$$


Com a finalidade de eliminar possíveis médias infinitas, introduzimos a seguinte notação:

$$
\bar{N}_{k}^{(n)}=\min \left(\bar{N}_{k}, n\right)
$$

que sustituida em (D.2) produz:

$$
\bar{N}_{k}^{(n)} \leq \min \left(n, \bar{N}_{11}+\bar{N}_{12}+\cdots+\bar{N}_{1 k}\right) \leq \bar{N}_{11}^{(n)}+\cdots+\bar{N}_{1 k}^{(n)}
$$

onde $\bar{N}_{1 i}^{(n)}=\min \left(n, \bar{N}_{1 i}\right)$. Seja $k>1$ um número arbitrário que satisfaz a condição do lema. Por (D.2) temos:

$$
\mathbb{E}\left[\bar{N}_{1}^{(n)}\right] \leq \sum_{l=1}^{k-1} P\left(t_{l-1}<S_{k} \leq t_{l}\right) l+\sum_{l=k}^{\infty} P\left(t_{l-1}<S_{k} \leq t_{l}\right)\left[l+\mathbb{E}\left[\bar{N}_{l-k+1}^{(n)}\right]\right]
$$

O primeiro somando $\sum_{l=1}^{k-1} P\left(t_{l-1}<S_{k} \leq t_{l}\right) l$ indica que, dado que em $t_{0^{-}}$há um componente para ser servido, para algum $k$ fixado, ocorre pelo menos uma renovação se o k-ésimo componente é reparado antes da $(k-1)$-ésima demanda.

O segundo somando: $\sum_{l=k}^{\infty} P\left(t_{l-1}<S_{k} \leq t_{l}\right)\left[l+\mathbb{E}\left[\bar{N}_{l-k+1}^{(n)}\right]\right]$ indica que se não ocorre nenhuma renovação até a $(k-1)$-ésima demanda, depois que se completa o $k$-ésimo serviço, o número de passos necessarios para uma renovação será $\bar{N}_{l-k+1}$, com $k \leq l<\infty$ Assim, temos de (D.3) e (D.4) que:

$$
\begin{aligned}
\mathbb{E}\left[\bar{N}_{1}^{(n)}\right] & \leq \sum_{l=1}^{\infty} P\left(t_{l-1}<S_{k} \leq t_{l}\right) l+\sum_{l=k}^{\infty} P\left(t_{l-1}<S_{k} \leq t_{l}\right) \mathbb{E}\left[\bar{N}_{l-k+1}^{(n)}\right] \\
& \leq \sum_{l=1}^{\infty}\left(\alpha_{k, l-1}-\alpha_{k l}\right) l+\sum_{l=k}^{\infty}\left(\alpha_{k, l-1}-\alpha_{k l}\right)(l-k+1) \mathbb{E}\left[\bar{N}_{1}^{(n)}\right] \\
& =1+\sum_{l=1}^{\infty} \alpha_{k l}+\mathbb{E}\left[\bar{N}_{1}^{(n)} \sum_{l=k-1}^{\infty} \alpha_{k l}\right. \\
& =\frac{1+\sum_{l=1}^{\infty} \alpha_{k l}}{1-\sum_{l=k-1^{\prime}}^{\infty} \alpha_{k l}}
\end{aligned}
$$

então, se $n \rightarrow \infty$, temos:

$$
\mathbb{E}\left[\bar{N}_{1}\right] \leq \frac{1+\sum_{l=1}^{\infty} \alpha_{k l}}{1-\sum_{l=k-1}^{\infty} \alpha_{k l}}
$$


Usamos o procedimento anterior para calcular $\mathbb{E}\left[\bar{N}_{0}\right]=\mathbb{E}[\bar{N}]$ :

$$
\begin{aligned}
\mathbb{E}[\bar{N}] & \leq P\left(t_{0}<S_{1} \leq t_{1}\right)+\sum_{l=1}^{\infty} P\left(t_{l}<S_{k} \leq t_{l+1}\right)\left[l+1+\mathbb{E}\left[\bar{N}_{l}\right]\right] \\
& \leq\left(1-\alpha_{1}\right)+\sum_{l=1}^{\infty}\left(\alpha_{l}-\alpha_{l+1}\right)\left[l+1+l \mathbb{E}\left[\bar{N}_{1}\right]\right] \\
& =\alpha_{0}+\left[1+\mathbb{E}\left[\bar{N}_{1}\right]\right] \sum_{l=1}^{\infty} \alpha_{l} \\
& =1+\left[1+\mathbb{E}\left[\bar{N}_{1}\right]\right] \alpha
\end{aligned}
$$

usando (D.5), temos:

$$
\begin{aligned}
\mathbb{E}[\bar{N}] & \leq 1+\alpha\left[1+\frac{1+\sum_{l=1}^{\infty} \alpha_{k l}}{1-\sum_{l=k-1}^{\infty} \alpha_{k l}}\right] \\
& =1+\frac{\alpha \sum_{l=1}^{k-2} \alpha_{k l}}{1-\sum_{l=k-1}^{\infty} \alpha_{k l}}
\end{aligned}
$$

$\operatorname{como} \sum_{l=1}^{k-2} \alpha_{k l} \leq k$, concluímos:

$$
\mathbb{E}[\bar{N}] \leq 1+\frac{\alpha k}{1-\sum_{l=k-1}^{\infty} \alpha_{k l}}
$$

e o lema está provado. 


\section{Apêndice E}

\section{Processo de renovação a tempo}

\section{discreto}

Considerando as notações do capítulo 2 e as probabilidades associadas com a ocorrência do evento $E=\left\{\right.$ renovação no instante $\left.t_{n}^{-}\right\}, \pi_{n}$ e $p_{n}$ relacionam-se de maneira similar à relação que existe entre $f_{i j}(n)$ e $p_{i j}(n)$ numa cadeia de Markov onde $f_{i j}(n)$ indica a probabilidade da primeira passagem do estado $i$ para o estado $j$ no instante $n$ e $p_{i j}(n)$ denota a probabilidade que começando no estado $i$, o sistema entra no estado $j$ no $n$-ésimo instante. (Ver [2], pag 110-112). Encontramos que:

$$
p_{n}=\sum_{l=1}^{n} \pi_{l} p_{n-l}
$$

Assim, dados os $\pi_{n}$, podemos fácilmente computar as probabilidades de (E.1):

$p_{1}=\pi_{1}$

$p_{2}=\pi_{1} p_{1}+\pi_{2} p_{0}$

$p_{3}=\pi_{1} p_{2}+\pi_{2} p_{1}+\pi_{3} p_{0}$

e assím sucessivamente, onde $p_{0}=1$. 
Se por outro lado, conhecemos os valores de $p_{n}$, usamos:

$$
\pi_{n}=p_{n}-\sum_{l=1}^{n-1} \pi_{l} p_{n-l}
$$

para computar os $\pi_{n}$.

Podemos calcular as probabilidades $\pi_{n}$ de $p_{n}$ usando (E.1) ou $p_{n}$ de $\pi_{n}$ usando (E.2), mas, uma forma elegante de computar estas probabilidades é por meio das funções geradoras de probabilidade. Para $|z| \leq 1$, sejam

$$
\begin{gathered}
p(z)=\sum_{n=0}^{\infty} p_{n} z^{n} e \\
\pi(z)=\sum_{n=1}^{\infty} \pi_{n} z^{n},
\end{gathered}
$$

as funções geradoras de probabilidade de $\left(p_{n}\right)_{n \geq 0}$ e $\left(\pi_{n}\right)_{n \geq 1}$, respectivamente.

\section{Teorema Auxiliar 1}

As funções geradoras de probabilidade $p(z)$ e $\pi(z)$ estão relacionadas pela fórmula:

$$
p(z)=\frac{1}{1-\pi(z)}
$$

ou alternativamente,

$$
\pi(z)=1-\frac{1}{p(z)}
$$

PROVA

A função geradora $p(z)$ pode ser escrita como:

$$
p(z)=z^{0} p_{0}+\sum_{n=1}^{\infty} p_{n} z^{n}
$$

sustituíndo em (E.1) temos:

$$
p(z)=1+\sum_{n=1}^{\infty} z^{n} \sum_{l=1}^{n} \pi_{l} p_{n-l}
$$


Para o lado direito de (E.6), pelo Teorema de Fubini, temos:

$$
\begin{aligned}
\sum_{n=1}^{\infty} z^{n} \sum_{l=1}^{n} \pi_{l} p_{n-l} & =\sum_{l=1}^{\infty} \sum_{n=l}^{\infty} \pi_{l} z^{n} p_{n-l} \\
& =\sum_{l=1}^{\infty} \sum_{n=l}^{\infty} z^{l} \pi_{l} z^{n-l} p_{n-l} \\
& =\left(\sum_{l=1}^{\infty} z_{l} \pi_{l}\right)\left(\sum_{n=0}^{\infty} z^{n} p_{n}\right) \\
& =\pi(z) p(z)
\end{aligned}
$$

sustituíndo (E.7) em (E.6) temos:

$$
p(z)=1+\pi(z) p(z)
$$

e o teorema está demonstrado. 


\section{Referências Bibliográficas}

[1] A. Solovyev B. Gnedenko Y. Beliaev, Mèthodes mathématiques en théorie de la fiabilité, Ed. Mir - Moscou, 1972.

[2] L.C. Chiang, An introduction to stochastic processes and their aplications, Krieger Publishing Company-New York, 1980.

[3] W. Feller, Introduction to probability and its aplications, Wiley - New York, 1966.

[4] I.B Gertsbakh, Statistical reliability theory, Marcel Dekker, INC, 1989.

[5] D. Gross e C.M. Harris, Fundamentals of queueing theory, Wiley-New York, 1974.

[6] Sheldom Ross, Applied probability models with optimization aplications, Holden Day, 1970.

[7] A.D Solovyev, Reliability and queueing theory, Engineering Cybernetics (1970). 